\title{
Transporte de sedimentos en suspensión en los principales ríos del Caribe colombiano: magnitud, tendencias y variabilidad
}

\author{
Juan Camilo Restrepo-López*, Juan Carlos Ortiz-Royero, Luis Otero-Díaz, Silvio Raúl Ospino-Ortiz \\ Grupo de Física Aplicada - Océano y Atmósfera, Departamento de Física, Universidad del Norte, Barranquilla, Colombia
}

\begin{abstract}
Resumen
Se analizaron series mensuales del transporte de sedimento en suspensión de siete ríos del Caribe colombiano, con el fin de proporcionar un estimativo actualizado, detectar los cambios recientes y los patrones de variabilidad del transporte de sedimento en suspensión. En su conjunto, estos ríos transportan un promedio de $\sim 146,3$ x10 $\mathrm{t}$ $\mathrm{a}^{-1}$ de sedimentos en suspensión hacia el litoral Caribe colombiano. El mayor aporte proviene del río Magdalena, con 142,6 x $10^{6} \mathrm{t} \mathrm{a}^{-1}$, que corresponden a $38 \%$ del total de los sedimentos en suspensión estimados para el litoral Caribe. La tasa anual del transporte de sedimentos en suspensión de estos sistemas fluviales ascendió hasta 36 \% entre 2000 y 2010. El análisis espectral basado en la transformada de ondícula (wavelet) evidenció lapsos de intensa variabilidad entre los periodos 1987-1990 y 1994-2002, en los cuales las principales componentes oscilatorias aparecieron de manera simultánea. Las bandas anual, semianual y 'cuasi-decadal' aparecieron como las principales componentes oscilatorias en la variabilidad del transporte de sedimento en suspensión, mientras que las bandas ‘cuasibienal' e interanual constituyeron una fuente de variabilidad de segundo orden. Se considera que los mecanismos forzadores climáticos y oceanográficos asociados con las oscilaciones detectadas mediante el análisis espectral definen un marco de variabilidad de mediano a largo plazo para el transporte de sedimentos en suspensión.
\end{abstract}

Palabras clave: transporte de sedimentos en suspensión, mar Caribe, variabilidad hidrológica, análisis espectral basado en la transformada de ondícula.

\section{Suspended sediment load in Colombian Caribbean Rivers: Magnitude, trends and variability}

\begin{abstract}
The data on the monthly average of suspended sediment load from seven rivers in northern Colombia (Caribbean alluvial plain) draining into the Caribbean Sea were analysed to quantify the magnitudes, to estimate long-term trends, and to evaluate the variability patterns. These rivers deliver an average of $\sim 146.3 \times 10^{6} \mathrm{t} \mathrm{yr}^{-1}$ of suspended sediments to the Colombian Caribbean littoral. The largest sediment supply is provided by the Magdalena River, with a mean suspended sediment load of $142.6 \times 10^{6} \mathrm{t} \mathrm{yr}^{-1}$, which is $38 \%$ of the total fluvial discharge estimated for the Caribbean littoral. Between 2000 and 2010, the annual suspended sediment load of these rivers increased to 36\%. The wavelet spectral analyses highlighted periods of intense variability between the periods of 1987-1990 and 19942002, where the major oscillation processes appeared simultaneously. The semi-annual, annual and quasi-decadal bands were the main factors controlling the suspended sediment load variability of these fluvial systems, whereas the quasi-biennial and interannual bands constituted second order sources of variability. The climatic/oceanographic drivers associated to the oscillations identified through the wavelet spectral analyses defined a medium/long term framework of variability for the suspended sediment load.
\end{abstract}

Key words: Suspended sediment load, Caribbean Sea, hydrologic variability, wavelet spectral analyses.

\section{Introducción}

Los sedimentos fluviales son un componente esencial de los ambientes acuáticos de gran importancia para los organismos vivos, pues desempeñan un papel fundamental en la estabilidad de los cuerpos de agua y los sistemas riberinos. Por consiguiente, los cambios en las tasas de transporte de sedimento en suspensión pueden causar impactos significativos en estos sistemas, así como en los ambientes estuarinos y deltaicos. Se ha demostrado que estos cambios incluso podrían tener efectos de mayor alcance que incluyen la transformación de los ecosistemas costeros de plataforma y la disminución de las poblaciones de peces (Yang, et al., 2003; Fan \& Huang, 2005; Le, et al., 2007; Wang, et al., 2007, 2010; Gao, et al., 2011).

\footnotetext{
*Correspondencia:

Juan Camilo Restrepo, restrepocj@uninorte.edu.co

Recibido: 19 de junio de 2015

Aceptado: 19 de octubre de 2015
} 
En Colombia la estimación del transporte de sedimentos, así como el estudio de los procesos que lo controlan, es incipiente, aunque se destaca la cuantificación del transporte de sedimentos en los principales sistemas fluviales del país (Restrepo \& Kjerfve, 2000, 2004), el análisis de los factores naturales y antrópicos que controlan la producción y el transporte de sedimentos en la cuenca del rio Magdalena (Restrepo \& Restrepo, 2005; Restrepo, et al., 2006; Restrepo \& Syvitski, 2006), y la construcción de modelos estadísticos para estimar el transporte de sedimento en suspensión en cuencas andinas (Restrepo \& Restrepo, 2005; Kettner, et al., 2010). Estos trabajos han destacado la relevancia de los flujos de sedimentos de los ríos colombianos en los balances globales de aporte de sedimentos a los océanos (Restrepo \& Kjerfve, 2000, 2004), la complejidad de los procesos naturales involucrados en la dinámica de los sedimentos en suspensión (Restrepo \& Restrepo, 2005; Restrepo, et al., 2006), y la importancia de los aportes fluviales en la progradación y arquitectura de los deltas colombianos, a pesar de la aparición de procesos destructivos como la subsidencia y el tectonismo (Restrepo \& López, 2008). No obstante, la mayoría de estos análisis se hicieron con series de tiempo cortas ( $<20$ años), recolectadas hasta mediados de la década de 1990. Por lo tanto, es poco probable que estos primeros estimativos reflejen los cambios naturales recientes, así como aquellos inducidos por la intervención antrópica. Por ejemplo, para finales de la década de 1990, aproximadamente $30 \%$ de los bosques de la región Caribe colombiana habían sido transformados en zonas de cultivos agrícolas y de ganadería extensiva, lo que convirtió a esta zona en un foco de deforestación (Etter, et al., 2006). En este sentido, Restrepo, et al. (2014) han señalado que entre 1998 y 2000 se presentó un cambio en los patrones hidrológicos de los ríos del Caribe colombiano caracterizado por el fortalecimiento de la señal oscilatoria cuasi-decadal, la superposición de los principales componentes oscilatorios, y el incremento pronunciado de los caudales después del año 2000, cuando los promedios anuales se incrementaron hasta $17 \%$ con respecto a los promedios de largo plazo. Los impactos de estos cambios sobre las tasas de transporte de sedimentos de los ríos de la región Caribe colombiana no han sido explorados; tampoco se han analizado sus cambios recientes, tendencias y patrones de variabilidad.

La determinación de las tendencias y patrones de variabilidad en las series de tiempo del transporte de sedimentos en suspensión constituye una técnica fundamental para comprender la importancia relativa de los cambios climáticos y las perturbaciones antrópicas, así como sus complejas interacciones. Las series de tiempo de largo plazo son esenciales para entender la dinámica del transporte de sedimentos en suspensión a escala de cuenca $\mathrm{y}$ en la posterior estimación de los flujos de nutrientes y sustancias biogeoquímicas (Liquete, et al., 2009; Slattery \& Phillips, 2009). En este trabajo se analizan de nuevo las series de transporte de sedimentos en suspensión en siete de los principales ríos del Caribe colombiano con el fin de (i) proporcionar un estimativo actualizado del transporte de sedimento en suspensión hacia el litoral Caribe colombiano; (ii) detectar cambios en las tasas de transporte de sedimento en suspensión durante los últimos 25 años, y (iii) identificar los patrones de variabilidad del transporte de sedimentos en suspensión en diferentes escalas de tiempo.

\section{El Caribe colombiano: contexto físico general}

\section{Aspectos fisiográficos}

La planicie Caribe colombiana está localizada en la región más septentrional de Suramérica. Se extiende desde la selva tropical del Darién, en la frontera colombo-panameña, hasta la península de La Guajira en el este y las estribaciones de la cordillera de los Andes en el sur (Figura 1). El Caribe colombiano está conformado por extensas planicies con alturas inferiores a $100 \mathrm{~m}$, serranías y mesetas con alturas entre 200 y $1.000 \mathrm{~m}$ en el suroeste (serranías de Abibe, San Jerónimo y Ayapel) y en el noreste (serranía de Macuira), y una de las montañas costeras más altas del mundo, la Sierra Nevada de Santa Marta, con alturas hasta de 5.000 m (Figura 1). Las planicies están dominadas por ecosistemas de sabana utilizados principalmente para la agricultura y el pastoreo. En las planicies y en las mesetas también pueden verse bosques tropicales secos y húmedos dependiendo de las condiciones de temperatura y precipitación. Las zonas montañosas están caracterizadas por una transición progresiva de bosque basal (1.000 - 1.200 m.s.n.m.) a bosque andino (1.000 - 4.000 m.s.n.m.), páramos (3.000 - 4.800 m.s.n.m.), y zona de glaciares permanentes en la Sierra Nevada de Santa Marta (>4.700 m.s.n.m.) (IDEAM, 1998). En la década de 1990 aproximadamente $30 \%$ de los bosques de la planicie del Caribe habían sido transformados en zonas de cultivos agrícolas y de ganadería extensiva. Sin embargo, se estima que durante los últimos años la tasa de deforestación en esta región ha disminuido de forma significativa (Etter, et al., 2006).

La oscilación meridional de la zona de convergencia intertropical (ZCIT) controla el ciclo hidrológico anual de la planicie Caribe colombiana y determina dos estaciones húmedas. La primera estación se extiende desde mayo hasta junio, cuando la ZCIT se desplaza hacia el norte. La segunda, más intensa, se extiende desde septiembre hasta noviembre, cuando la ZCIT se desplaza hacia el sur. Sin embargo, se han identificado algunos patrones locales como resultado del efecto de las corrientes de chorro del oeste y del norte (es decir, chorros del Chocó y San Andrés), y los efectos orográficos inducidos por la cordillera de los Andes y la Sierra Nevada de Santa Marta (Bernal, et al., 2006; Poveda, 2004). Las masas de aire húmedo provenientes del océano Pacifico son transportadas por advección por la corriente de chorro del Chocó, que las hace ascender rápidamente a lo largo de las vertientes de la cordillera Occidental. Este proceso favorece su convección profunda y fortalece 


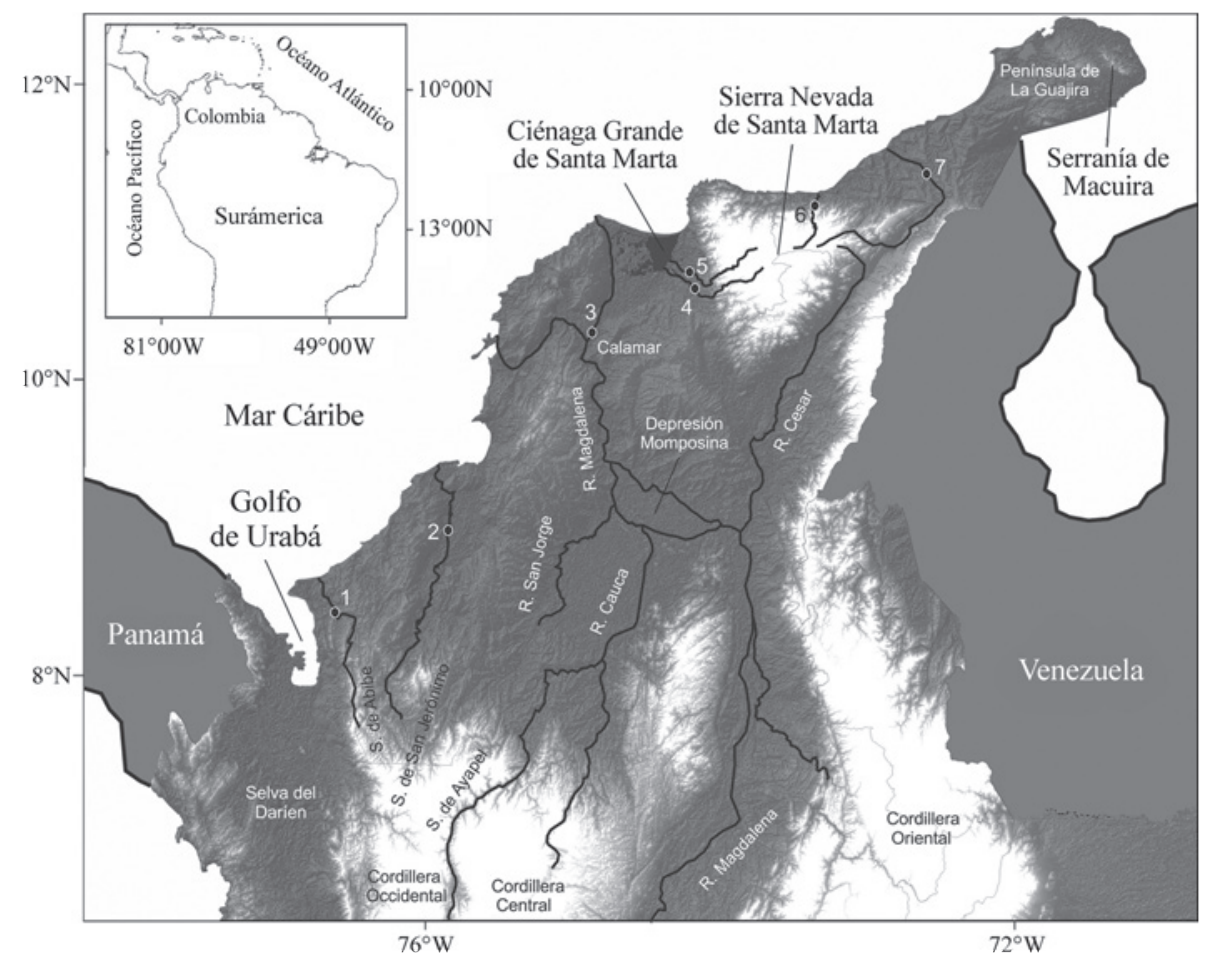

Figura 1. (A) Planicie Caribe colombiana, incluidos sus accidentes geográficos y ríos principales, y sus respectivas estaciones hidrológicas. 1. Mulatos, 2. Sinú, 3. Magdalena, 4. Fundación, 5. Aracataca, 6. Palomino y 7. Ranchería.

los sistemas convectivos de mesoescala que, a su vez, conducen a altas tasas de precipitación (Poveda \& Mesa, 2004). La interacción entre los vientos alisios del noreste y los cinturones de baja presión ( $<900 \mathrm{hPa}$ ) localizados en la latitud $13^{\circ} \mathrm{N}-14^{\circ} \mathrm{N}$, favorece la formación de la corriente de chorro de San Andrés. Esta corriente de chorro causa una divergencia de humedad en el noroeste de Suramérica, la cual fortalece el ascenso de masas de aire a lo largo de las vertientes de la Sierra Nevada de Santa Marta, pero ocasiona fuertes corrientes superficiales de viento y baja humedad en la península de la Guajira (Bernal, et al., 2006). En el Caribe colombiano la temporada húmeda de mayor intensidad coincide con el periodo de mayor magnitud del chorro del Chocó, mientras que la estación seca coincide con la máxima intensidad del chorro de San Andrés. Por consiguiente, el sector occidental de la planicie del Caribe, cerca del bosque húmedo tropical del Darién, y la Sierra Nevada de Santa Marta exhiben tasas de precipitación y temperaturas medias anuales de $>2.000 \mathrm{~mm} \mathrm{a}^{-1} \mathrm{y}<20{ }^{\circ} \mathrm{C}$, respectivamente. En contraste, las zonas bajas $(<100 \mathrm{~m})$ son más secas, con tasas de precipitación por debajo de $1.000 \mathrm{~mm} \mathrm{a}^{-1}$, y más cálidas, con temperaturas medias anuales de $>27^{\circ} \mathrm{C}$ (Mesa, et al., 1997). En escalas mayores de tiempo, las anomalías significativas en los patrones hidrológicos están asociadas a ambas fases del fenómeno de El Niño-Oscilación del Sur (ENOS). La fase cálida del ENOS (El Niño) genera un incremento en la temperatura media del aire, un descenso en la humedad del suelo y en el índice de vegetación (Normalized Difference Vegetation Index, NDVI), y, en consecuencia, se presenta una disminución significativa en las tasas de precipitación. Por el contrario, las anomalías ocasionadas durante la fase fría del ENOS (La Niña) conducen a abundantes e intensas precipitaciones (Poveda, et al., 2001; Poveda, 2004). De acuerdo con Restrepo \& Kjerfve (2004), los ríos del Caribe colombiano experimentan una fuerte variación estacional en sus caudales, por lo que generalmente se obtiene una proporción hasta de 5 a 10 veces cuando se comparan los caudales mínimos y máximos mensuales. Estos autores también indican que la variabilidad asociada al ENSO puede ser igualmente grande y, usualmente, se calcula una proporción hasta de 2 a 4 veces cuando se comparan los caudales mínimos y máximos anuales.

Los ríos que drenan la planicie Caribe colombiana se originan en nacimientos localizados en la cordillera de los Andes (Mulatos, Sinú, Magdalena) y la Sierra Nevada de Santa Marta (Aracataca, Fundación, Palomino, Ranchería) (Figura 1). Los ríos Mulatos y Sinú nacen en el Nudo de Paramillo, en donde la cordillera Occidental se bifurca en las serranías de Abibe, San Jerónimo y Ayapel. El río Mulatos, localizado sobre el cinturón del Sinú, drena una meseta en la serranía de Abibe a lo largo de 115 km en dirección surnorte antes de desembocar en el mar Caribe. El río Sinú tiene un área de drenaje de $14,7 \times 10^{3} \mathrm{~km}^{2}$, que comprende una zona con pendientes pronunciadas en su nacimiento, un valle aluvial formado por las serranías de Abibe y San 
Jerónimo, así como una extensa llanura aluvial en la que, además, existe una compleja red de ciénagas. Este río tiene una extensión de $415 \mathrm{~km}$ desde su nacimiento, a una altura de $3.960 \mathrm{~m}$, hasta su desembocadura en el delta de Tinajones en el mar Caribe.

El río Magdalena es el mayor sistema fluvial de la planicie Caribe colombiana, con una extensión de $1.540 \mathrm{~km}$ y un área de drenaje de $257,4 \times 10^{3} \mathrm{~km}^{2}$. Su nacimiento está localizado en la cordillera Central (sur de Colombia, $1^{\circ} 45^{\prime} \mathrm{N}, 76^{\circ} 30^{\prime} \mathrm{O}$ ) a una altura de $3.600 \mathrm{~m}$. El río se dirige hacia el norte a lo largo del Valle del Magdalena, entre las cordilleras Central y Oriental, y tras abandonar este valle se dirige al oeste y entra en una depresión tectónica activa conocida como la Depresión Momposina. En esta depresión tectónica, que actúa como un delta interior, se han estimado altas tasas de sedimentación (2,1-3,0 $\mathrm{mm} \mathrm{a}^{-1}$ ) (van der Hammen, 1986). En esta zona, el río Magdalena tiene un curso con meandros y recibe los aportes fluviales de los ríos Cesar, Cauca y San Jorge (Restrepo \& Kjerfve, 2004). Una vez cruza la Depresión Momposina, el río se dirige hacia el norte hasta Calamar, en donde se forman dos canales distributarios. El Canal del Dique, uno de estos distributarios, se dirige hacia el noroeste hasta desembocar en la bahía de Cartagena, mientras que el principal distributario continúa su curso hacia Barranquilla, a lo largo del extremo occidental del cinturón de San Jacinto, en donde desemboca en el mar Caribe.

Los ríos que nacen en la Sierra Nevada de Santa Marta drenan cuencas montañosas de baja extensión $\left(<5.000 \mathrm{~km}^{2}\right)$, caracterizadas por pendientes pronunciadas y planicies de inundación estrechas. Excepto en el caso del río Ranchería, que drena extensas planicies a lo largo de la península de La Guajira. Los ríos Aracataca y Fundación drenan la vertiente occidental de la Sierra Nevada de Santa Marta y desembocan en la Ciénaga Grande de Santa Marta. Esta ciénaga tiene una conexión directa con el mar Caribe en el extremo oriental de la barra de Salamanca. Los ríos Palomino y Ranchería drenan la vertiente oriental de la Sierra Nevada de Santa Marta, en sentido suroeste - noreste, y desembocan directamente en el mar Caribe (Figura 1).

\section{Contexto geológico}

El marco geológico regional está determinado por el movimiento e interacción de las placas de Suramérica y Nazca, el arco volcánico de Panamá, y el segmento occidental de la placa oceánica del Caribe (Trenkamp, et al., 2002; Cediel, et al., 2003). Los regímenes de esfuerzos de estas placas se distribuyen principalmente entre los bloques Norandino y Panamá-Chocó. El bloque Norandino comprende los Andes septentrionales y el Caribe colombo-venezolano y se mueve hacia el nororiente a una velocidad de $6 \mathrm{~mm} \mathrm{a}^{-1}, \mathrm{y}$ colisiona con el bloque Panamá-Chocó a una tasa de $25 \mathrm{~mm}$ $\mathrm{a}^{-1}$ (Trenkamp, et al., 2002).
Las serranías de Abibe, San Jerónimo y Ayapel hacen parte de los cinturones del Sinú y San Jacinto, que se formaron como resultado de la compresión causada por la colisión del bloque Panamá-Chocó contra la placa Sudamericana, y al actuar sobre los sedimentos cenozoicos acumulados en el margen continental dieron originen a una franja de rocas altamente deformadas (Duque-Caro, 1987). El cinturón del Sinú, que se extiende al occidente de la falla del Sinú, está compuesto por rocas sedimentarias con edades desde el Eoceno medio hasta el Oligoceno tardío (48-23 Ma). Por su parte, el cinturón de San Jacinto, limitado por las fallas del Sinú y Romeral, está conformado por rocas sedimentarias con edades entre el Cretáceo y el Mioceno (65-3,5 Ma) (Ingeominas, 2003). El río Sinú representa el límite entre los cinturones del Sinú y San Jacinto, por lo que su valle aluvial, formado por un evento diastrófico ocurrido durante el Pleistoceno-Holoceno, presenta un marcado control tectónico (Duque-Caro, 1979). La configuración actual de la cuenca del Magdalena es el resultado de procesos evolutivos que datan del Paleozoico (450 Ma) (Mojica \& Franco, 1990). Las fallas de Romeral y Santa Marta-Bucaramanga constituyen los límites cordilleranos que debido a la orogénesis del Plioceno modelan el curso y los depósitos sedimentarios de la cuenca del Magdalena (Martínez \& López, 2005). Como resultado de la interacción de las placas tectónicas y de la acreción de los cinturones de Sinú y San Jacinto en el Caribe, se formó el arco de Magangué y la cuenca del Plato-San Jorge, en donde posteriormente se asentó la Depresión Momposina (Duque-Caro, 1984).

La Sierra Nevada de Santa Marta es un macizo rocoso aislado de las demás cadenas montañosas que conforman el bloque Norandino y flanqueado por las cuencas sedimentarias de Plato-San Jorge y Cesar-Ranchería (Montes, et al., 2010). Los ríos Aracataca y Fundación se localizan sobre la cuenca Plato-Cesar, la cual contiene secuencias marinas no deformadas del Eoceno superior al Plioceno y se encuentra en contacto tectónico con la Sierra a lo largo de la falla Santa Marta-Bucaramanga (Montes, et al., 2010). Por su parte, los ríos Palomino y Ranchería se localizan sobre la cuenca Cesar-Ranchería, una cuenca no fallada formada hacia el suroeste en continuidad con la Sierra Nevada de Santa Marta, que preserva una cuña clástica de sedimentos marino-continentales que datan del Cretáceo tardío al Neógeno (Montes, et al., 2010).

\section{Datos y métodos}

Este estudio analizó los datos históricos de transporte de sedimentos en suspensión en siete cuencas del litoral Caribe colombiano con diferentes características climáticas y topográficas. Se analizaron datos mensuales en las estaciones más cercanas a la desembocadura en el mar Caribe (Tabla 1), con el fin de, (1) estimar los aportes fluviales de sedimento hacia la zona litoral, (2) determinar y cuantificar tendencias en las series históricas, y (3) determinar las periodicidades y los patrones de variabilidad interanual. Se obtuvieron 
Tabla 1. Nombres de los ríos y estaciones de aforo evaluadas en este estudio, con la localización y registro histórico de los datos de transporte de sedimentos en suspensión (TSS)

\begin{tabular}{|c|c|c|c|c|c|}
\hline \multirow[t]{2}{*}{ Río } & \multirow[t]{2}{*}{ Estación de aforo } & \multicolumn{3}{|c|}{ Localización } & \multirow{2}{*}{$\begin{array}{c}\text { Registro } \\
\text { (TSS) }\end{array}$} \\
\hline & & Elevación (m.s.n.m.) & Longitud & Latitud & \\
\hline \multicolumn{6}{|l|}{ Ríos andinos } \\
\hline 1. Mulatos & Pueblo Bello & 84 & $76^{\circ} 310$ & $8^{\circ} 12 \mathrm{~N}$ & $1985-2007$ \\
\hline 2. Sinú & Cotoca Abajo & 5 & $75^{\circ} 510$ & $9^{\circ} 13 \mathrm{~N}$ & $1984-2010$ \\
\hline 3. Magdalena & Calamar & 8 & $74^{\circ} 550$ & $10^{\circ} 15 \mathrm{~N}$ & $1972-2010$ \\
\hline \multicolumn{6}{|c|}{ Ríos de la Sierra Nevada } \\
\hline 4. Fundación & Fundación & 55 & $74^{\circ} 110$ & $10^{\circ} 31 \mathrm{~N}$ & $1982-2009$ \\
\hline 5. Aracataca & Puente Ferrocarril & 37 & $74^{\circ} 110$ & $10^{\circ} 35 \mathrm{~N}$ & $1976-2009$ \\
\hline 6. Palomino & Puente Carretera & 30 & $73^{\circ} 340$ & $11^{\circ} 14 \mathrm{~N}$ & $1976-2009$ \\
\hline 7. Ranchería & Hacienda Guamito & 76 & $72^{\circ} 370$ & $11^{\circ} 10 \mathrm{~N}$ & $1980-2006$ \\
\hline
\end{tabular}

Nota. La numeración de los ríos corresponde a la señalada en la Figura 1; ríos andinos: nacimientos en la cordillera de los Andes; ríos de la Sierra Nevada: = nacimientos en la Sierra Nevada de Santa Marta

datos del periodo comprendido entre 1972 y 2010 (Tabla 1), los cuales fueron proporcionados por el Instituto de Hidrología, Meteorología y Estudios Ambientales de Colombia (IDEAM). La selección de los ríos y sus respectivas estaciones de monitorización se basó en las siguientes condiciones: (1) la estación de aforo debía estar localizada en la parte más baja de la cuenca de drenaje, y (2) el registro hidrológico debía ser superior a 20 años. (1) La serie de tiempo medida en la desembocadura de una cuenca se considera una señal valiosa, que integra las ganancias y pérdidas del balance sedimentario (es decir, la producción de sedimentos, su transporte y depositación); por consiguiente, estas series de tiempo constituyen un sustituto adecuado para estimar el transporte de sedimentos hacia la zona costera (Walling \& Fang, 2003; Walling, 2009; Syvitski \& Kettner, 2011). Por lo tanto, en este trabajo se asumió que estos valores representaban una aproximación a la cantidad de sedimentos transportados hasta las zonas deltaicas y estuarinas formadas en las desembocaduras de estos ríos. Estas zonas actúan como filtros que atrapan una gran proporción de los sedimentos fluviales (Dyer, 1995, 1997). (2) Definiendo $T$ como la extensión total del registro hidrológico, la frecuencia de corte () y el cono de influencia (edge effects) () delimitan la significación estadística de las señales identificadas a partir de un análisis de series de tiempo (Shumway \& Stoffer, 2004). La extensión de las series de transporte de sedimento en suspensión varía entre 23 y 39 años (Tabla 1 ).

Se aplicaron pruebas de continuidad y homogeneidad a los promedios mensuales de transporte de sedimentos en suspensión. Se analizaron las series de tiempo mediante el test de Mann-Kendall (gradual trend test) para identificar y cuantificar tendencias hidrológicas (Yue, et al., 2002); se estableció la correlación cruzada entre los valores mensuales del transporte de sedimentos en suspensión (TSS) y las anomalías mensuales del índice de oscilación del sur (IOS) para cuantificar los efectos del ENSO sobre las descargas fluviales, y se hizo un análisis de la transformada continua de ondícula (continuous wavelet transform, CWT) para determinar y estimar las periodicidades y patrones de variabilidad (Labat, 2005; Shumway \& Stoffer, 2004). Los datos de las anomalías mensuales del IOS se obtuvieron de la National Oceanographic and Atmospheric Administration - Climate Prediction Center, NOAA) (NOAA, 2012).

El test de Mann-Kendall (Man-Kendall Test, MKT) es un prueba estadística no paramétrica basada en el rango que se utiliza para detectar y evaluar la significación de tendencias monotónicas en series de tiempo (Yue, et al., 2002). Las pruebas no paramétricas no asumen ninguna forma especial para la función de distribución de datos, por lo tanto, se considera que son más apropiadas para datos que no se ajustan a una distribución normal y constituyen una de las técnicas más idóneas para identificar tendencias lineales en datos ambientales, específicamente en el análisis de datos de descarga fluvial (García \& Mechoso, 2005; Milliman, et al., 2008; Pasquini \& Depetris, 2007; Yue, et al., 2002; Zhang, et al., 2008). Si se asume como hipótesis nula que no existe tendencia en la serie de tiempo, y se considera que los datos son independientes e idénticamente distribuidos, puede calcularse una variable estandarizada $(Z)$ para verificar la hipótesis nula. La hipótesis nula es rechazada para un nivel de significancia $\alpha$ si $Z>Z_{(1-\alpha / 2)}$, en donde $Z_{(1-\alpha / 2)}$ es el valor estándar de una distribución normal con probabilidad de $\alpha / 2$ (Yue, et al., 2002). Considerando que los datos de TSS posteriores al año 2000 no habían sido analizados previamente en los ríos del Caribe colombiano, y que el MKT puede considerarse como un indicador de cambio hidrológico, se aplicó dicha prueba a los valores del periodo 2000-2010 con el fin de evaluar la influencia de los valores recientes en la tendencia de largo plazo y detectar cambios hidrológicos recientes usando esta prueba estadística como un sustituto (Dai, et al., 2009; Restrepo, et al., 2014). 
La transformada continua de ondícula (CWT) se usó para examinar las series de tiempo empleando funciones generalizadas de base local (mother wavelets) que se desplazan y estiran simultáneamente en el dominio de la frecuencia y el tiempo (Torrence \& Compo, 1998). Por lo tanto, la CWT ofrece ventajas significativas con respecto a métodos más tradicionales como la transformada rápida de Fourier porque permite la localización de señales de frecuencia en una escala de tiempo. Una de las ventajas de la ondícula es que es independiente de la escala, por lo que resulta útil para analizar series de tiempo que contienen funciones no estacionarias de diferentes frecuencias. El CWT se aplicó a los datos 'desestacionalizados' para estimar periodicidades y patrones de variabilidad y para distinguir las oscilaciones temporales en las series de tiempo, determinando, además, la intermitencia de cada proceso de escala-tiempo. También se aplicó un análisis de frecuencia de potencias. Se calculó la potencia de un espectro continuo de ondícula (Global Wavelet Spectrum) en el tiempo para una banda de frecuencia específica mediante la integración de dicho espectro con base en el rango de frecuencias (Labat, 2005). Esta prueba permite aislar varias bandas de frecuencia y obtener información detallada acerca de la potencia de una periodicidad específica a través del dominio del tiempo. El espectro de la ondícula se promedió en el tiempo para cuantificar las escalas dominantes de los procesos hidrológicos subyacentes, lo que permitió la estimación de la distribución de la señal de varianza entre las diferentes escalas (Torrence \& Compo, 1998). La ventaja decisiva del espectro global de ondícula radica en que puede estimar eficientemente las escalas características de los procesos de largo plazo (Labat, et al., 2005). Para identificar las oscilaciones significativas se calculó un intervalo de confianza de $95 \%$ para los contornos y el cono de influencia siguiendo la metodología propuesta por Torrence \& Compo (1998). La aplicación del CWT a series de TSS ha permitido la detección de oscilaciones de diferente escala asociadas a diversos mecanismos forzadores hidroclimáticos (Zhang, et al., 2008; Liu, et al., 2013).

\section{Resultados}

Aportes de sedimentos en suspensión al litoral Caribe: magnitud y tendencias de largo plazo

La tasa media mensual de transporte de sedimentos en suspensión (TSS) de los ríos que drenan la planicie Caribe colombiana varió entre $0,1 \times 10^{3} \mathrm{t} \mathrm{d}^{-1}$ y 390,8 x10 $\mathrm{t} \mathrm{d}^{-1}$ (Tabla 2). La diferencia entre la media mensual y el valor máximo mensual fue hasta de un orden de magnitud, mientras que la diferencia entre los valores máximos y mínimos mensuales fue hasta de tres órdenes de magnitud (Tabla 2). Las tasas más altas de TSS se registraron en los ríos con las mayores áreas de drenaje ( $\left.>14,0 \times 10^{3} \mathrm{~km}^{2}\right)$, mientras que los ríos con áreas de drenaje pequeñas $\left(<5,0 \times 10^{3} \mathrm{~km}^{2}\right)$ presentaron las mayores variaciones mensuales del TSS (Tabla 2). En su conjunto, estos ríos transportaron en promedio $\sim 146,3 \times 10^{6} \mathrm{t} \mathrm{a}^{-1}$ de sedimentos en suspensión hacia el litoral Caribe. La mayor contribución correspondió al río Magdalena, con 142,6 X10 t $\mathrm{a}^{-1}$, que representa el $\sim 97 \%$ de la descarga total promedio de sedimentos en suspensión aportada por estos ríos.

Las series interanuales mostraron una tendencia decreciente en los ríos Mulatos, Sinú, Fundación y Aracataca, mientras que los ríos Magdalena, Palomino y Ranchería exhibieron una tendencia ascendente (Figura 2). Sin embargo, el análisis MKT indicó que sólo la tendencia exhibida por el río Sinú fue significativa, con un nivel de confianza de $95 \%$ (Tabla 3). La magnitud del cambio del TSS por unidad de tiempo, calculada como la relación entre la pendiente de Sen y la tasa media mensual de TSS, fue mayor en los ríos Mulatos y Sinú (Figura 3). Después del año 2000, con la excepción del río Mulatos, todas las tendencias se hicieron más acentuadas o presentaron un cambio en la dirección de su pendiente (Figura 2 y Tabla 4). En este último periodo, solo el río Magdalena exhibió una tendencia significativa, con un nivel de confianza de $95 \%$. Aunque la mayoría de los ríos no mostraron tendencias significativas en sus registros, el TSS anual de todos estos sistemas fluviales cambió hasta en $36 \%$ entre 2000 y 2010 (Tabla 4). Por ejemplo, el valor medio anual de TSS registrado en el Sinú antes del $2000\left(3,68 \times 10^{6} \mathrm{t} \mathrm{a}^{-1}\right)$, fue $36 \%$ mayor comparado

Tabla 2. Área de drenaje (A), altura del nacimiento, tasa media mensual de transporte de sedimentos en suspensión (TSS), transporte de sedimento en suspensión - máximo mensual $\left(\mathrm{TSS}_{\max }\right.$ ), transporte de sedimento en suspensión - mínimo mensual (TSS min $_{\text {) }}$, de los ríos que drenan la planicie Caribe colombiana

\begin{tabular}{|c|c|c|c|c|c|}
\hline Río & $A\left(10^{3} \mathbf{k m}^{2}\right)$ & Nacimiento (m.s.n.m.) & $\operatorname{TSS}\left(\mathbf{x 1 0 ^ { 3 }} \mathbf{t ~ d}^{-1}\right)$ & $\mathrm{TSS}_{\max }\left(\mathrm{x10}^{3} \mathrm{t} \mathrm{d}^{-1}\right)$ & 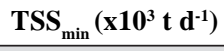 \\
\hline Mulatos & 0,01 & 1.118 & 0,469 & 6,24 & 0,001 \\
\hline Sinú & 14,73 & 3.960 & 8,620 & 31,90 & 0,265 \\
\hline Magdalena & 257,43 & 3.600 & 390,869 & 1564,00 & 46,830 \\
\hline Fundación & 1,87 & 2.986 & 0,244 & 3,13 & 0,003 \\
\hline Aracataca & 0,93 & 4.408 & 0,100 & 1,18 & 0,001 \\
\hline Palomino & 0,68 & 4.785 & 0,130 & 1,51 & 0,002 \\
\hline Ranchería & 4,23 & 3.700 & 0,287 & 9,74 & 0,001 \\
\hline
\end{tabular}



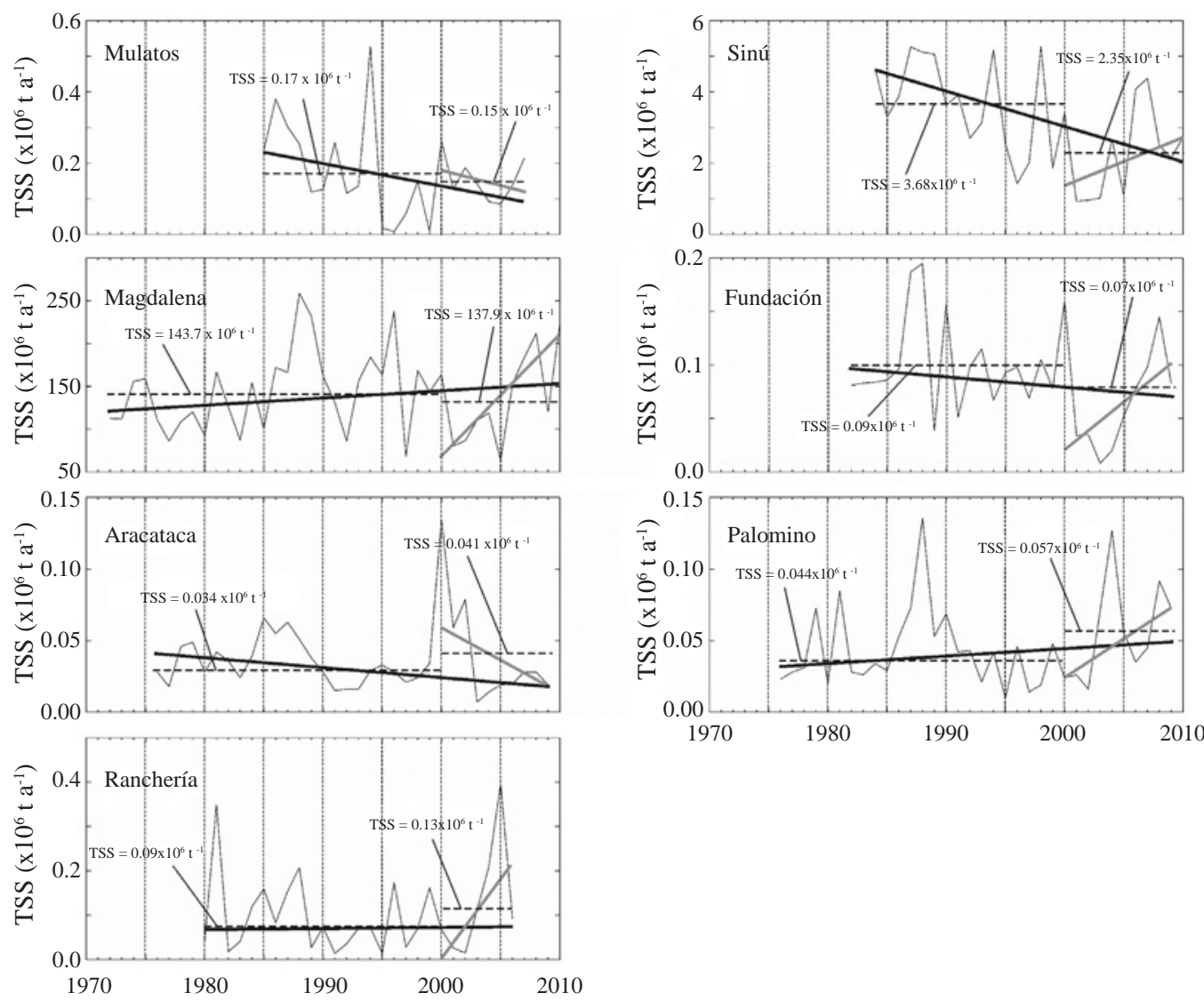

Figura 2. Transporte de sedimentos en suspensión (TSS): media anual (línea delgada), tendencia de largo plazo (línea negra gruesa), tendencia para el periodo 2000-2010 (línea gris gruesa) y promedio interanual para periodos de interés (línea negra punteada) en los ríos de la planicie Caribe colombiana

Tabla 3. Resultados de los análisis del test de Mann-Kendall y la pendiente de Sen para las series de tiempo de transporte de sedimentos en suspensión anual de los ríos de la planicie Caribe colombiana

\begin{tabular}{|c|c|c|c|c|c|c|c|}
\hline \multirow[t]{2}{*}{ Río } & \multirow[t]{2}{*}{ Estación de aforo } & \multicolumn{5}{|c|}{ Test de Mann - Kendall } & \multirow{2}{*}{$\begin{array}{c}\text { Pendiente de Sen } \\
\left(\mathbf{x}^{10^{6}} \mathrm{t} \mathrm{a}^{-1} \mathrm{a}^{-1}\right)\end{array}$} \\
\hline & & Primer año & Último año & No. de años & Test Z & $\mathbf{p}$ & \\
\hline Mulatos & Pueblo Bello & 1985 & 2007 & 23 & $-1,27$ & n.s. & $-0,006$ \\
\hline Sinú & Cotoca Abajo & 1984 & 2010 & 27 & $-2,38$ & $<0,05$ & $-0,099$ \\
\hline Magdalena & Calamar & 1972 & 2010 & 39 & 0,99 & n.s. & 0,846 \\
\hline Fundación & Fundación & 1982 & 2009 & 28 & $-0,85$ & n.s. & $-0,001$ \\
\hline Aracataca & Puente Ferrocarril & 1976 & 2009 & 34 & $-1,39$ & n.s. & 0,000 \\
\hline Palomino & Puente Carretera & 1976 & 2009 & 34 & 0,95 & n.s. & 0,001 \\
\hline Ranchería & Hacienda Guamito & 1976 & 2006 & 27 & 0,25 & n.s. & 0,000 \\
\hline
\end{tabular}

n.s. $=$ estadísticamente no significativo

con el TSS medio anual experimentado durante el intervalo 2000-2010 (2,35 x10 $\left.\mathrm{t} \mathrm{a}^{-1}\right)$. Por su parte, el Ranchería pasó de transportar 0,09 x10 $\mathrm{t} \mathrm{a}^{-1}$ de sedimentos en suspensión en promedio antes del 2000 a registrar un TSS promedio de $0,13 \times 10^{6} \mathrm{t} \mathrm{a}^{-1}$ entre 2000 y 2007. El menor cambio se registró en el Magdalena, con un descenso de $\sim 4 \%$ en la tasa media anual de TSS (Tabla 4).
Efectos preliminares de El Niño-Oscilación del Sur (ENSO)

Los resultados de un análisis de correlación cruzada entre el TSS mensual y las anomalías mensuales del índice de oscilación del sur (IOS), calculado para cuantificar preliminarmente los efectos del ENSO en las series de tiempo de TSS, se presentan en la Figura 4. Entre 1950 y 2010, se presentaron 11 y 8 eventos de El Niño y La Niña de magnitud 
moderada a fuerte, respectivamente (Figura 4). En general, los altos valores mensuales de TSS y las anomalías positivas del IOS mostraron correlaciones entre bajas y moderadas; en consecuencia, no se presentó una relación sistemática y consistente entre estos dos parámetros. Por ejemplo, las tasas de TSS anormalmente altas ( $>2$ desviaciones estándar) que se presentaron en la mayoría de los ríos durante el periodo 1988 - 1989, coincidieron con una larga e intensa fase fría del ENSO (La Niña) que se extendió desde abril de 1988 a julio de 1989 (Figura 4). Sin embargo, algunos valores excepcionalmente altos de TSS ( $>4$ desviaciones estándar) se registraron en años normales o durante la fase cálida del ENSO (El Niño), por ejemplo, en los ríos Mulatos (6

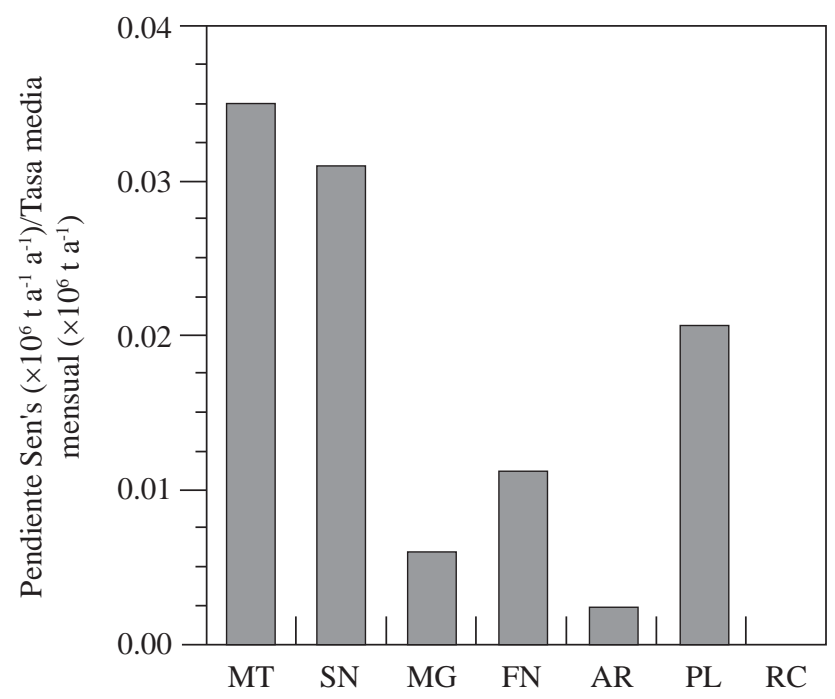

Figura 3. Magnitud del cambio en el transporte de sedimentos en suspensión (TSS) por unidad de tiempo (cociente entre la pendiente de Sen y la tasa media mensual de TSS en los ríos Mulatos (MT), Sinú (SN), Magdalena (MG), Fundación (FN), Aracataca (AR), Palomino (PM), y Ranchería (RC) desviaciones estándar) en 1995, Aracataca (6 desviaciones estándar) en 1986 y 2002, y Ranchería (8 desviaciones estándar) en 2006 (Figura 4). Las mayores correspondencias entre las anomalías IOS y el TSS se observaron en 1988, 1999-2000, 2008, y 2010. Las fuertes anomalías positivas del IOS registradas en estos periodos coincidieron con TSS anormalmente altos. En general, las mayores correlaciones entre el TSS y el IOS se encontraron para el río Magdalena (Figura 4).

Las desviaciones negativas de TSS suelen ser de poca magnitud, generalmente de menos de dos desviaciones estándar, mientras que las anomalías negativas del IOS pueden llegar hasta valores de 4. En este caso, estas desviaciones y anomalías negativas mostraron coincidencia pero no proporcionalidad y, por lo tanto, exhibieron poca correlación (Figura 4). Por ejemplo, el evento de El Niño de 1991-1992, que fue uno de los más desastrosos para Colombia, coincidió con un descenso en las tasas de TSS en la mayoría de estos ríos. Sin embargo, la magnitud de tale descensos no fue proporcional a la anomalía negativa del IOS (Figura 4).

\section{Periodicidades: oscilaciones de corto y largo plazo}

Las oscilaciones semianuales fueron intermitentes y relativamente dispersas en la mayoría de los ríos. El río Mulatos registró un componente de 6 meses, visible entre los periodos 1986-1988, 1994-1995, y 2000-2010, mientras que el río Fundación exhibió este componente entre 1985-1991 y 2007-2010. Los ríos Magdalena, Aracataca, Palomino y Ranchería mostraron fluctuaciones de este componente entre 1975-1985, 1985-1992, y 2000-2010 (Figura 5). La dinámica temporal de la oscilación anual también varió entre los diferentes ríos. Esta señal fue estacionaria para el río Sinú hasta 1999, y reapareció con intensidad a partir de 2006. En los demás ríos la componente anual fue una señal cuasi-continua de magnitud comparable, con cortos periodos

Tabla 4. Ríos de la planicie Caribe colombiana: resultados del MKT, pendiente de Sen’s, y tasa media anual de transporte de sedimentos en suspensión para dos periodos seleccionados (Pre-2000 y Post-2000).

\begin{tabular}{|c|c|c|c|c|c|c|c|c|c|c|}
\hline \multirow[t]{3}{*}{ Rio } & \multicolumn{5}{|c|}{ Pre - 2000} & \multicolumn{5}{|c|}{ Post - 2000} \\
\hline & \multicolumn{3}{|c|}{ Mann - Kendall Test } & \multirow{2}{*}{$\begin{array}{c}\text { Pendiente Sen's } \\
\left(\mathbf{x 1 0}^{6} \mathrm{ta}^{-1} \mathrm{a}^{-1}\right)\end{array}$} & \multirow{2}{*}{$\frac{\text { TSS }}{\left(\times 10^{6} t^{-1}\right)}$} & \multicolumn{3}{|c|}{ Mann - Kendall Test } & \multirow{2}{*}{$\begin{array}{c}\text { Pendiente Sen's } \\
\left({\left.\mathrm{x} 10^{6} \mathrm{t} \mathrm{a}^{-1} \mathrm{a}^{-1}\right)}\right.\end{array}$} & \multirow{2}{*}{$\frac{\text { TSS }}{\left({\left.\mathrm{x} 10^{6} \mathrm{t} \mathrm{a}^{-1}\right)}^{-1}\right.}$} \\
\hline & Primer año & Último año & Test Z & & & Primer Año & Último Año & Test Z & & \\
\hline Mulatos & 1985 & 1999 & $-2.18^{*}$ & -0.020 & 0.179 & 2000 & 2007 & n.d. & -0.009 & 0.156 \\
\hline Sinú & 1984 & 1999 & $-1.85^{* *}$ & -0.169 & 3.680 & 2000 & 2010 & 1.40 & 0.139 & 2.355 \\
\hline Magdalena & 1972 & & 1.64 & 1.552 & 143.702 & 2000 & 2010 & $2.02 *$ & 14.290 & 137.900 \\
\hline Fundación & 1982 & 1999 & 0.30 & 0.001 & 0.099 & 2000 & 2009 & 1.25 & 0.009 & 0.071 \\
\hline Aracataca & 1976 & 1999 & -1.22 & -0.001 & 0.034 & 2000 & 2009 & -0.72 & -0.005 & 0.041 \\
\hline Palomino & 1976 & 1999 & -0.22 & 0.000 & 0.044 & 2000 & 2009 & 1.61 & 0.006 & 0.057 \\
\hline Ranchería & 1976 & 1999 & -0.36 & 0.000 & 0.096 & 2000 & 2006 & n.d. & 0.036 & 0.131 \\
\hline
\end{tabular}

* Significancia para un nivel de confianza del $95 \%$

** Significancia para un nivel de confianza del $90 \%$;

n.d. = no disponible, porque no existe el número suficiente de datos para calcular el estimador $Z$ 

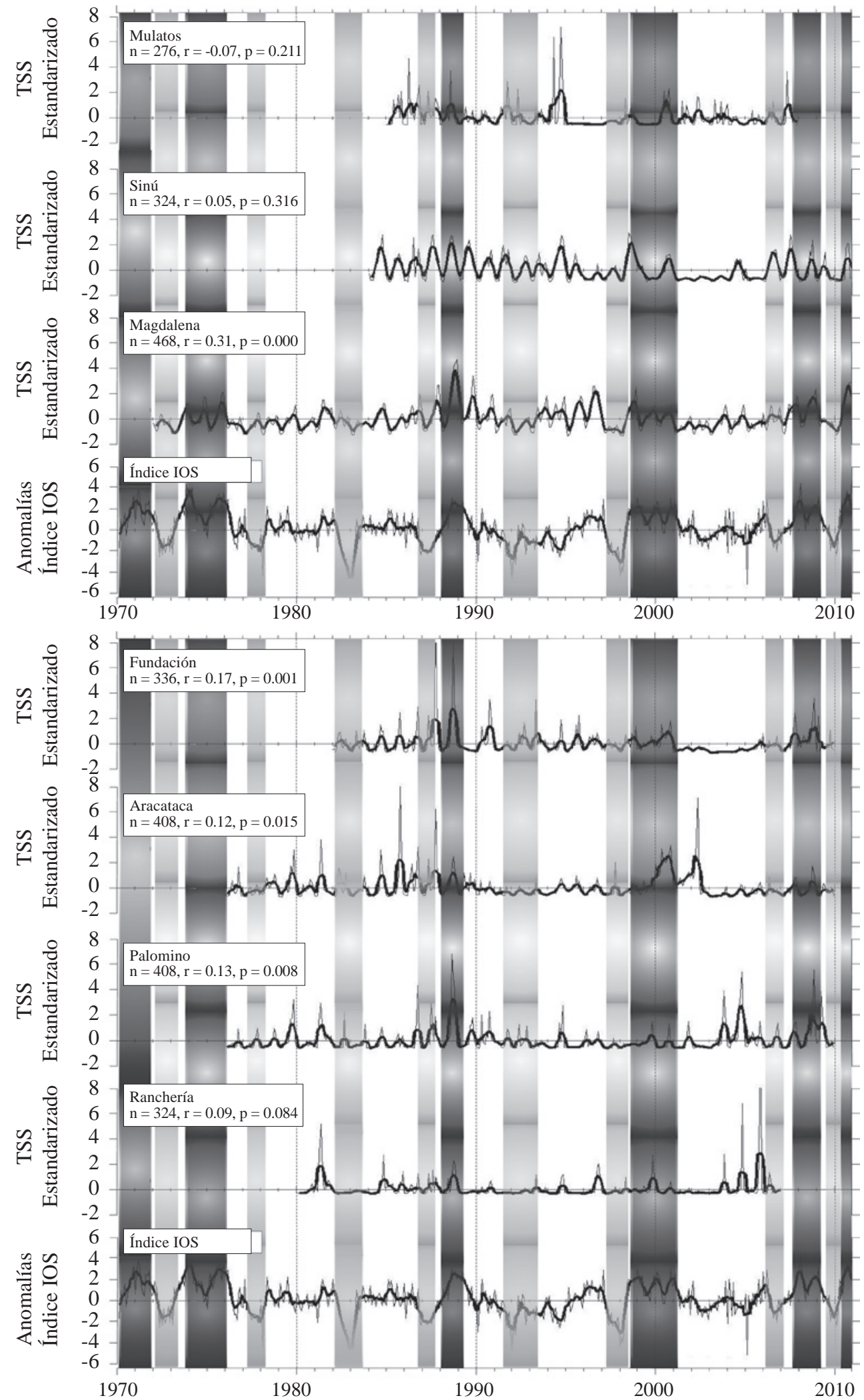

Figura 4. Valores mensuales estandarizados de transporte de sedimento en suspensión (TSS) y anomalías del índice de oscilación sur (IOS) (líneas delgadas); media móvil de cinco meses sobrepuesta sobre las series de tiempo (línea negra gruesa). Los recuadros gris y gris claro representan las fases fría y cálida del fenómeno ENSO, respectivamente. Los estadísticos de la correlación cruzada entre las tasas mensuales de TSS y las anomalías mensuales del IOS se observan en el recuadro superior izquierdo. 

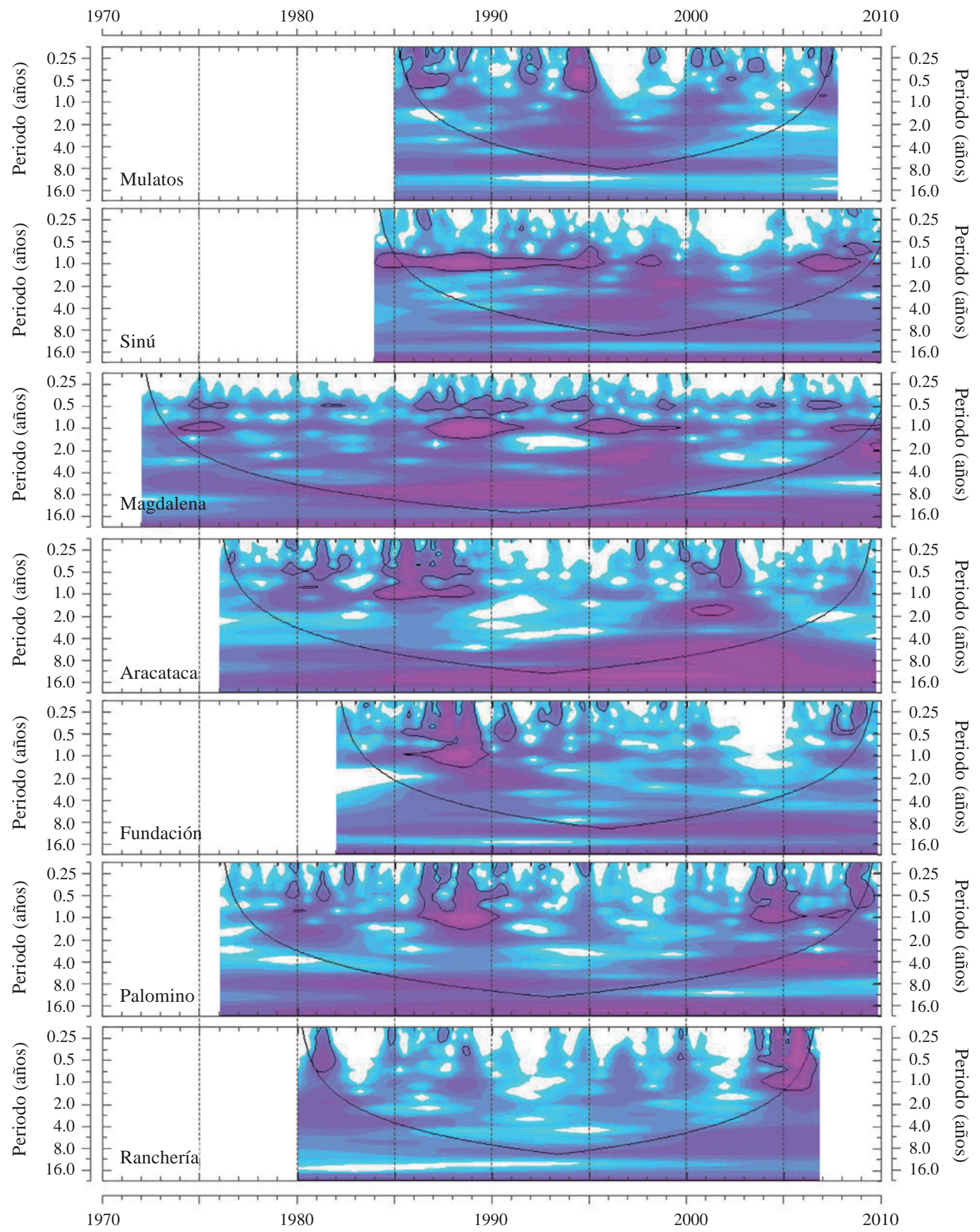

Figura 5. Transformada continua de ondícula (CWT) para el transporte de sedimentos en suspensión de los ríos de la planicie colombiana. El color purpura oscuro corresponde a valores altos y el claro a valores bajos de los coeficientes de la transformada (potencia). El contorno negro grueso delimita las zonas con un nivel de confianza de $95 \%$ contra un ruido rojo AR(1); el cono de influencia en donde los efectos de borde no son despreciables se señala con una línea negra delgada. 
de alta potencia. Por ejemplo, los ríos Aracataca, Fundación y Palomino exhibieron un corto periodo de intensa actividad en el intervalo 1985-1989 (Figura 5). En el CWT también se destacó un componente cuasi-bienal en la variabilidad del TSS, el cual fue más intenso en el río Mulatos durante el intervalo 1995-2003, en el Sinú entre 1997 y 2003, en el Magdalena entre los periodos 1987-2000 y 2009-2010, en el Aracataca durante el periodo 1998-2005, en el Fundación entre 1989-1993, en el Palomino entre los periodos 19771983, 1987-1992 y 2008-2010, y en el Ranchería en los intervalos 1980-1983 y 2005-2006 (Figura 5).

Las fluctuaciones interanuales del TSS en estos sistemas fluviales se caracterizaron por un componente intermitente de 4 a 7 años. En el CWT del río Ranchería se destacó un componente de 4 a 5 años en los intervalos de 1980-1985 y 2000-2010, y un componente de 5 a 7 años entre 1995 y 2010. La componente de 4 a 5 años apareció en los ríos Mulatos, Sinú y Aracataca durante 1990-2000, 1994-1999, y 1995-2005, respectivamente, y se extendió desde 2000 a 2010 en el río Palomino. Por otra parte, la fluctuación de 5 a 7 años se presentó en el periodo de 1990-2002 en el río Sinú, de 1980-2007 en el río Aracataca, y de 1980-1995 en el río Palomino. En el CWT del TSS del río Magdalena se encontró una componente de 4 a 5 años entre 1997 y 2002, y una de 5 a 7 años entre 1983 y 2000, con una potencia máxima durante el periodo 1985-1996 (Figura 5). Una oscilación cuasi-decadal (8-12 años) se registró en los ríos Magdalena, Aracataca y Palomino en 1985. Esta componente oscilatoria se extendió hasta 2010 en los dos primeros ríos y hasta 2005 en el último, y fue particularmente intensa a inicios de la década del 2000 en el rio Aracataca (Figura 5).

El CWT resaltó un periodo de intensa actividad en los periodos 1987-1990 y 1994-2002, en los cuales las principales componentes oscilatorias aparecieron de manera simultánea. Todos los ríos, excepto el Ranchería, exhibieron oscilaciones superpuestas de las señales de 0,5 a 1 año, 2 a 3 años, y 4 a 5 años durante el intervalo de 1987-1990, mientras que las oscilaciones de 1 año y 2 a 3 años coincidieron con la señal interanual en los ríos Mulatos, Sinú, Magdalena y Aracataca a lo largo del periodo 1994-2002 (Figura 5). Durante estos periodos se presentaron altos valores de TSS (Figura 4).

El análisis de potencia de frecuencia específica, que permite la recolección de información detallada sobre la potencia de una periodicidad a lo largo del tiempo, se hizo para las señales de 2 a 8 años y 8 a 12 años (Figura 6). El componente de 2 a 8 años se caracterizó por presentar oscilaciones menores y una oscilación dominante en la que se alcanzó la máxima potencia. Aunque estas oscilaciones dominantes no estuvieron en fase, mostraron una relativa proximidad en algunos ríos. Por ejemplo, en el río Magdalena el periodo de máxima oscilación se presentó en 1997, en el río Sinú en 1999, y en el río Aracataca en 2001. Los ríos Palomino y Ranchería mostraron su máxima oscilación alrededor de 2005 y 2006, respectivamente (Figura 6a). Debido al efecto del cono de influencia en el CWT, solo se analizó la variabilidad de la componente de 8 a 12 años en los ríos con una serie de tiempo mayor a 30 años (Tabla 1). En estos ríos, esta componente mostró una única gran oscilación, que se intensificó a partir de 1976 en el río Palomino y a partir de 1985 en los ríos Magdalena y Aracataca. El punto de máxima potencia de este componente oscilatorio se alcanzó en 1987 en el río Palomino, 1995 en el río Magdalena, y 2002 en el río Aracataca (Figura 6b).

El proceso de integración de los CWT con respecto al tiempo permite obtener el espectro global de ondícula, como se muestra en la Figura 7. En los ríos Magdalena, Fundación y Ranchería la banda anual y la semianual aparecen como las principales componentes oscilatorias. En escalas interanuales la componente de 4 a 7 años fue común en todos los ríos, excepto en el río Sinú, que exhibió una marcada oscilación anual (Figura 7). Esta banda interanual aparece como la oscilación dominante en el río Mulatos, mientras que constituyó una fuente de variabilidad de segundo orden en los demás ríos. En la mayoría de los ríos también se pudo establecer la oscilación cuasi-bienal como una fuente de segundo orden en la variabilidad del TSS. En los ríos Aracataca y Palomino la fluctuación de 8 a 12 años fue la principal componente oscilatoria (Figura 7). Las componentes oscilatorias mayores a un año no fueron significativas, por lo que deben interpretarse con prudencia. Sin embargo, esta información se considera de utilidad debido a que las señales identificadas están dentro del rango definido por la frecuencia de corte y el cono de influencia; la técnica de rellenado con ceros (zero padding) podría reducir la verdadera potencia de las oscilaciones de baja frecuencia, y el CWT aísla las señales ocultas en el ruido (y que no son reveladas mediante otras técnicas convencionales). En consecuencia, se considera que se requieren series de tiempo más extensas para probar la significación estadística de estas oscilaciones de baja frecuencia.

\section{Discusión}

\section{Transporte de sedimento en suspensión: magnitud y tendencias de largo plazo}

Los ríos evaluados en este estudio mostraron un TSS acumulado de 146,3 x10 $\mathrm{t} \mathrm{a}^{-1}$ (Tabla 2), que representa aproximadamente el $39 \%$ de la descarga total de sedimentos (378 x10 $\mathrm{a}^{-1}$ ) estimada para la zona litoral del mar Caribe (Park, 1999). El río Magdalena contribuyó con la mayor cantidad de estos aportes, con un TSS de 142,6 x106 $\mathrm{t} \mathrm{a}^{-1}$ (Tabla 2), que corresponde al $38 \%$ del total de los aportes de sedimentos en suspensión estimados para esta zona. Previamente, Restrepo \& Kjerfve (2004) habían indicado que el transporte combinado de 22 ríos del Caribe colombiano ascendía a $168 \times 10^{6} \mathrm{t} \mathrm{a}^{-1}$ de sedimentos en suspensión, y que entre ellos se destacaba el río Magdalena con una tasa de TSS de 143,9 x10 $\mathrm{t} \mathrm{a}^{-1}$. Otros valores de TSS reportados para el río Magdalena varían entre 133,1 x10 $\mathrm{t} \mathrm{a}^{-1}$ y 220 x10 

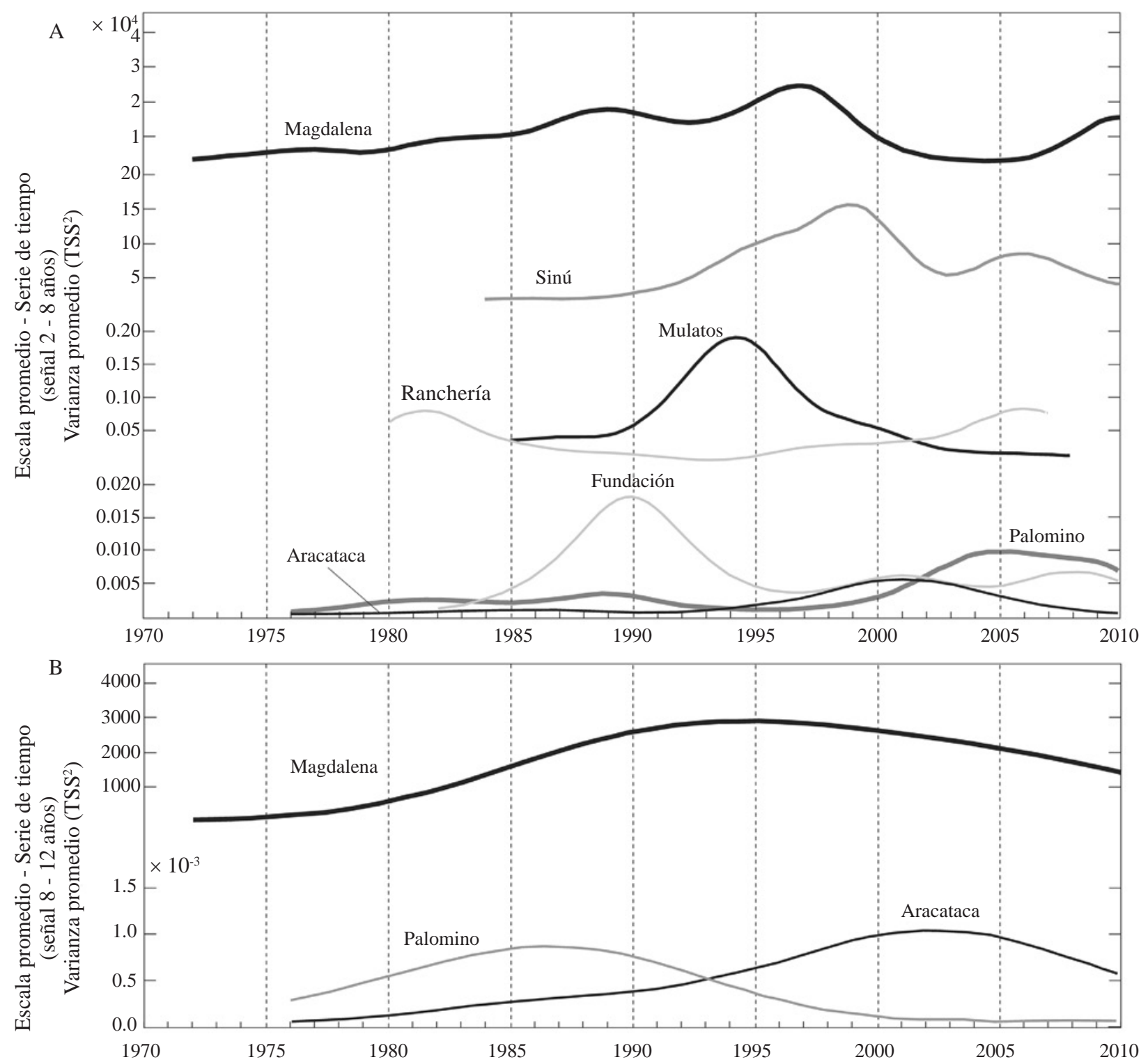

Figura 6. Transporte de sedimento en suspensión - análisis de potencia de frecuencias específicas: varianza promedio para la señal de (a) 2-8 años y (b) 8-12 años

t a ${ }^{-1}$ (Milliman \& Meade, 1983; Alvarado, 1992; Marín, 1992; Restrepo \& Kjervfe, 2000). No obstante, estos valores se calcularon a partir de las mediciones puntuales realizadas por NEDECO (1973) a principios de la década de 1970 (Milliman \& Meade, 1983), o mediante el análisis de series de tiempo con una longitud inferior a 20 años y con registros hasta 1995 (Marín, 1992; Restrepo \& Kjervfe, 2000, 2004). El conjunto de datos presentado en este estudio, aunque incluye un menor número de ríos comparado con el estudio de Restrepo \& Kjerfve (2004), comprende series de tiempo de mayor extensión (>22 años) e incluye registros recientes. Por lo tanto, debido a la extensión y cobertura temporal de las series analizadas, consideramos que los valores de TSS de este estudio representan un estimativo de largo plazo más confiable que los reportados previamente.
Los resultados del estudio confirman lo señalado previamente por Restrepo \& Kjerfve (2000) en cuanto a que el TSS del río Magdalena se encuentra entre los mayores a nivel mundial (Milliman \& Meade, 1983; Milliman \& Syvitski, 1992; Syvitski \& Kettner, 2011; Syvitski, 2011), y es de magnitud comparable al que se presenta en ríos con mayores áreas de drenaje (A), como, por ejemplo, el Orinoco (Venezuela) (TSS $=150 \times 10^{6} \mathrm{t} \mathrm{a}^{-1} ; \mathrm{A}=0,99 \times 10^{6}$ $\mathrm{km}^{2}$ ), el Paraná (Argentina) (TSS $=79 \times 10^{6} \mathrm{t} \mathrm{a}^{-1} ; \mathrm{A}=2,6$ $\mathrm{x} 10^{6} \mathrm{~km}^{2}$ ) y el Huanghe (China) (TSS $=150 \times 10^{6} \mathrm{t} \mathrm{a}^{-1}$; A $=0,77 \times 10^{6} \mathrm{~km}^{2}$ ) (Milliman \& Syvitski, 1992; Wang, et al., 2007, 2010). Esto significa que los flujos de sedimento del río Magdalena constituyen un elemento central en la progradación y arquitectura del delta del Magdalena (porción sub-aérea y sub-acuosa), en la morfodinámica 

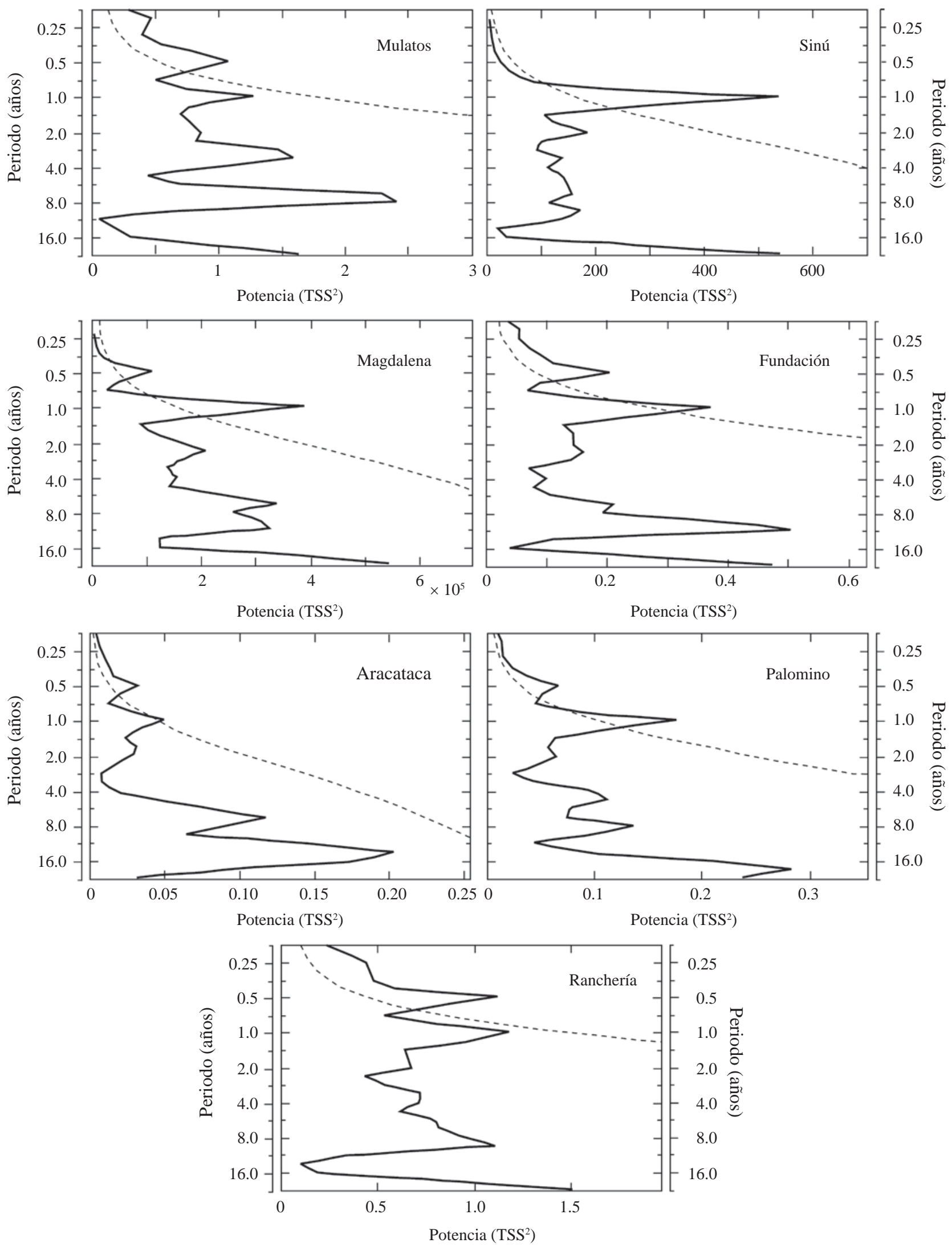

Figura 7. Transporte de sedimento en suspensión: espectro global de ondicula de los ríos evaluados. La línea punteada delimita las zonas con un nivel de confianza de $95 \%$ contra un ruido rojo AR(1). 
litoral y en la transferencia de nutrientes al mar Caribe. Aunque los demás ríos presentan tasas relativamente bajas de TSS $\left(<1,0 \times 10^{6} \mathrm{t} \mathrm{a}^{-1}\right)$ (Tabla 2), la presencia de procesos de migración de meandros y lagunas costeras (backwater) y de captura de sedimentos en deltas y estuarios sugiere que una gran proporción de los sedimentos se deposita aguas arriba de su desembocadura en el mar Caribe, por lo que, a pesar de sus bajas descargas, estos aportes fluviales desempeñarían un papel significativo a escala local en la estabilidad morfológica de deltas, estuarios y playas.

La relación observada entre el área de drenaje y la magnitud y variabilidad del TSS (Tabla 2) recalca dos aspectos ampliamente estudiados y aceptados: los ríos con una mayor área de drenaje presentan mayores tasas y una menor variabilidad en el TSS debido a la gran extensión de las áreas de fuente y a un mayor número de zonas disponibles para la acumulación de sedimentos, respectivamente, en tanto que en los ríos con una menor área de drenaje se presenta el efecto contrario debido al número relativamente bajo de áreas de fuente y de zonas de depositación de sedimentos (Walling, 1983,; Milliman \& Syvitski, 1992; Hovius, 1998; Harrison, 2000; Dearing \& Jones, 2003). Se ha demostrado que no existe continuidad en la transferencia de sedimentos desde las áreas de fuente hasta los sistemas fluviales, particularmente en cuencas con grandes áreas de drenaje en donde la acumulación de sedimentos puede presentarse en diferentes escalas espaciales y de tiempo (Meade, 1996; Walling, 1983). La ausencia de continuidad conduce a rezagos en el flujo de sedimentos entre las áreas de fuente y las áreas de depositación, localizadas principalmente en las grandes zonas de almacenamiento de depósitos aluviales y coluviales. Estos rezagos generalmente constituyen la principal fuente de desequilibrio entre la producción de sedimento y el transporte neto de sedimento en la desembocadura de un río. Una estimación de esta continuidad la proporciona el índice conocido como Sediment Delivery Ratio (SDR), que representa el porcentaje de la erosión total de la cuenca que es transportado hasta el mar como TSS (Walling, 1983). A su vez, se ha encontrado que el SDR y el área de drenaje tienen una relación inversamente proporcional, en la que el primero tiende a disminuir en la medida en que el área de captación se incrementa (Walling, 1983; Dearing \& Jones, 2003). En consecuencia, las cuencas con áreas de drenaje pequeñas $\left(A<5000 \mathrm{~km}^{2}\right)$ y altos relieves (como la mayoría de las analizadas en este estudio; Tabla 2), generalmente tienen una respuesta más rápida frente a los cambios ambientales y, por lo tanto, su variabilidad es mayor (Dearing \& Jones, 2003).

El TSS fluvial es un parámetro variable (Meybeck, et al., 2003; Morehead, et al., 2003). El coeficiente de variación anual del TSS de los ríos analizados en este estudio es mayor a 1 (Tabla 5), lo que indica que resulta más complejo detectar tendencias en los registros de TSS dada su inherente
Tabla 5. Valores promedio $(\bar{x})$, desviación estándar $(\sigma)$ y coeficiente de variación $(\mathrm{Cv})$ del transporte de sedimentos en suspensión de los ríos que drenan la planicie Caribe colombiana

\begin{tabular}{|c|c|c|c|}
\hline \multirow[t]{2}{*}{ Río } & \multicolumn{3}{|c|}{ Transporte de sedimento en suspensión } \\
\hline & $\bar{x}\left(x 10^{3} t^{-1}\right)$ & $\sigma\left(\mathbf{x} 10^{3} t_{d^{-1}}\right)$ & Cv (-) \\
\hline Mulatos & 0,469 & 0,807 & 1,721 \\
\hline Sinú & 8,620 & 8,031 & 0,932 \\
\hline Magdalena & 390,869 & 249,269 & 0,638 \\
\hline Fundación & 0,244 & 0,326 & 1,328 \\
\hline Aracataca & 0,100 & 0,132 & 1,325 \\
\hline Palomino & 0,130 & 0,203 & 1,557 \\
\hline Ranchería & 0,287 & 0,765 & 2,666 \\
\hline
\end{tabular}

variabilidad natural. Además, como resultado de su baja capacidad para amortiguar los efectos naturales o antrópicos y de sus SDR relativamente altos, las pequeñas cuencas exhiben mayores perturbaciones en sus regímenes de TSS $y$, en consecuencia, presentan una mayor variabilidad comparadas con los ríos que poseen grandes áreas de drenaje. De ahí la complejidad que implica detectar tendencias estadísticamente significativas en las series de TSS.

Además de la alta variabilidad natural del transporte de sedimentos, un registro de TSS que no presente una tendencia significativa y que, por lo tanto, pueda asumirse como estable, también podría indicar la inexistencia de cambios en el clima o en las características fisiográficas de la cuenca, así como la capacidad de la cuenca de modular dichos cambios como resultado del almacenamiento y una nueva movilización de sedimentos, de manera que los flujos de sedimento permanezcan esencialmente estables, o la interacción de cambios que generen efectos opuestos en el TSS (Walling \& Fang, 2003; Phillips, et al., 2004; Wang, et al., 2010). Teniendo en cuenta que por sus características fisiográficas, en la mayoría de estas cuencas se esperan altos valores de SDR, lo cual implica una menor capacidad de modulación, y que los resultados del análisis de tendencias y periodicidades del caudal hechos por Restrepo, et al. (2014) registraron un periodo de cambio hidrológico de escala regional entre 1998-2010, puede considerarse que la ausencia de tendencias significativas de TSS en estos ríos se debe fundamentalmente a la gran variabilidad natural y a los cambios con efectos opuestos en los procesos de transporte de sedimentos.

Por último, en el análisis se resaltó que después del 2000 la mayoría de las tendencias del TSS se hicieron más acentuadas o presentaron un cambio en la dirección de su pendiente, lo que refleja los cambios experimentados durante el periodo 2000-2010 en las tasas anuales de TSS, las cuales variaron hasta $36 \%$ durante este periodo (Figura 2 y Tabla 4). Algo similar se reportó para el caudal de algunos ríos del Caribe colombiano (Restrepo, et al., 2014). La aparición 
simultánea de estos cambios a lo largo de toda la planicie Caribe colombiana sugiere que el mecanismo de generación tiene una cobertura espacial de escala regional.

\section{Variabilidad del transporte de sedimento en suspensión}

Debido a la extensión de las series de tiempo de TSS de estos ríos, prácticamente ningún estudio había analizado previamente su relación con parámetros oceanográficos y climáticos de mesoescala como el ENSO. Solamente Restrepo \& Kjerfve (2000) habían señalado que el ENSO podía explicar hasta el $54 \%(\mathrm{r}=0,74)$ de la variabilidad interanual del TSS en el río Magdalena: altas tasas de TSS durante La Niña, mientras que durante El Niño se experimentaba el efecto contrario. Los resultados obtenidos en este estudio indicaron que los valores mensuales de TSS del río Magdalena y las anomalías del IOS presentaban una correlación entre moderada y baja (r=0,31) (Figura 4) y, en consecuencia el ENSO, asociado a la banda de oscilación interanual (Zhang, et al., 2008) constituye una fuente de variabilidad de segundo orden en el TSS. La principal diferencia radica en que los resultados de Restrepo \& Kjerfve (2000) se basaron en el análisis de correlación cruzada entre las series mensuales de anomalías en el IOS y el TSS filtradas o suavizadas, lo que llevó a estimar un coeficiente de correlación superior al esperado. El análisis espectral del CWT permite detectar un mayor número de señales no estacionarias en las series de tiempo $\mathrm{y}$, por lo tanto, evaluar su efecto en la variabilidad del TSS. En general, el efecto del ENSO fue mayor en los ríos Mulatos (Figura 7) y Magdalena (Figura 4). Considerando que la influencia del ENSO es mayor en las regiones del Pacífico y los Andes que en la región Caribe de Colombia (Mesa, et al., 1997; Poveda, et al., 2001) estos resultados pueden explicarse en términos de la proximidad del río Mulatos a la cuenca del océano Pacífico $(\sim 170 \mathrm{~km})$, y a que la mayor parte de la cuenca del Magdalena se encuentra localizada en la región Andina ( $83 \%$ ).

Los estudios sobre la variabilidad del TSS se han concentrado en evaluar el efecto de factores individuales como el nivel del mar, la escorrentía, la tectónica, el clima, la construcción de embalses y los cambios en el uso del suelo, o una combinación de estos factores (Milliman \& Syvitski, 1992; Hovius, 1998; Inman \& Jenkins, 1999; Harrison, 2000; Meybeck, et al., 2003; Morehead, et al., 2003; Walling \& Fang, 2003; Zhang, et al., 2008). Sin embargo, el transporte y la depositación de sedimentos responden simultáneamente a todos estos procesos, y otros más. Además, estos mecanismos forzadores están interrelacionados y los grados de libertad, así como las condiciones de frontera para la respuesta de un sistema, están condicionados por factores geológicos y litológicos (Inman \& Jenkins, 1999; Wang, et al., 2007, 2010; Slattery \& Phillips, 2009). Actualmente hay evidencia limitada en cuanto al efecto neto del cambio o variabilidad climática sobre el TSS de los ríos (Walling \& Fang, 2003; Walling, 2009), particularmente en el análisis de escala regional (Inman \& Jenkins, 1999; Liquete, et al., 2009; Slattery \& Phillips, 2009). Esta situación refleja la dificultad inherente a la separación de los efectos producidos por los cambios climáticos de aquellos generados por la variabilidad interanual de los ríos, y la necesidad de incluir otras fuentes no estacionarias en los análisis de series de tiempo de TSS.

Por ejemplo, aunque la tasa media de TSS del Sinú disminuyó 36 \% en el periodo 2000-2010, este cambio no necesariamente reflejó los efectos producidos por la operación del Embalse de Urrá I (2000) por varias razones: el TSS ya mostraba una tendencia decreciente entre 1970 y 2000, por el contrario, entre 2000 y 2010 el TSS presentó una tendencia creciente, y el marcado descenso en las tasas de TSS observadas en el Sinú a comienzos de la década del 2000 coincidió con las disminuciones experimentadas durante este mismo periodo en otros ríos, lo cual indica que estos cambios tuvieron un alcance de escala regional (Figura 2). Como se asume que los datos de TSS recolectados en la desembocadura reflejan el balance de los procesos e intervenciones que se presentan en una cuenca hidrográfica, estos resultados indican que en el Sinú otros efectos (es decir, cambios hidrológicos, cambios en el uso del suelo) prevalecen sobre la retención de sedimentos en el embalse de Urrá I. Por lo anterior, es necesario analizar los datos de estaciones localizadas en la parte alta y media de la cuenca para evaluar el efecto neto producido por la construcción de este embalse.

En la mayoría de estos ríos el análisis espectral del CWT evidenció la presencia simultánea de varias oscilaciones en periodos en los que se observaron altas tasas de TSS, como los ocurridos en 1987-1990, 1994-2002, y 2008-2010 (Figura 5). Este análisis también indicó que, aunque las oscilaciones dominantes del TSS no estuvieron en fase, en algunos de los ríos los periodos de máxima potencia tuvieron una relativa proximidad temporal (Figura 5). Además, al comparar en cada uno de los ríos los espectros globales de ondícula del TSS y del caudal (Restrepo, et al., 2014), se observan resultados similares en cuanto a la detección de las principales componentes oscilatorias (excepto en el caso del río Mulatos) (Figura 8). Estos resultados nos permiten sugerir que los mecanismos forzadores que controlan la variabilidad del caudal (los mencionados por Restrepo, et al., 2014; la migración de la ZCIT; los procesos climáticos y oceanográficos de baja frecuencia como la oscilación decadal del Pacífico, o la del Atlántico tropical norte, y el ENSO), también determinan la variabilidad del TSS. Las diferencias en la fase y la magnitud de las oscilaciones dominantes (Figura 5 y 7) se explican por las características fisiográficas y litológicas de las cuencas y su respectiva capacidad para regular los cambios climáticos o antrópicos.

El SDR y la litología también pueden explicar las diferencias que se observaron al comparar la aparición y la magnitud de las oscilaciones en los espectros de TSS (Figura 5). Se 

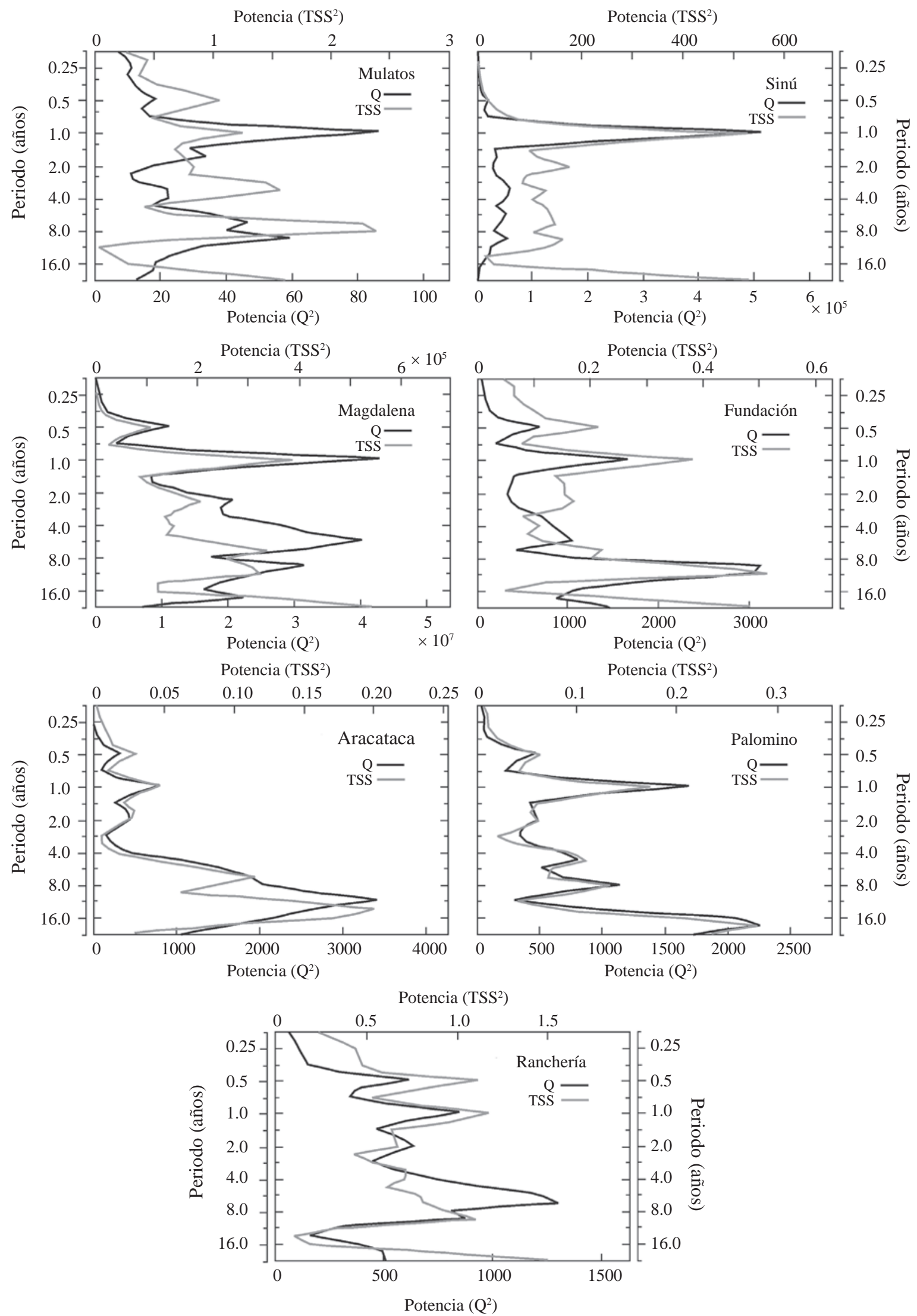

Figura 8. Comparación entre el espectro global de ondícula del transporte de sedimento en suspensión (TSS) y el del caudal, obtenido por Restrepo, et al. (2014) 
ha estimado que la intervención humana en los procesos naturales está incrementando las tasas de erosión global, probablemente por un factor de dos (Milliman \& Syvitski, 1992). Sin embargo, el impacto de estas actividades puede ser mucho menos directo en cuencas con grandes áreas de drenaje, debido a que solo una baja proporción de la cuenca se ve afectada por dichas actividades. Por lo tanto, el progresivo impacto de la intensificación en el uso del suelo solo genera cambios graduales en los flujos de sedimentos y una proporción significativa de los sedimentos transportados desde la parte alta de la cuenca podría depositarse y acumularse antes de alcanzar la desembocadura (Walling \& Fang, 2003; Wilkinson, 2005). El SDR proporciona una estimación del efecto de estos procesos sobre la transferencia de sedimentos. Además, el tipo de roca también puede explicar las diferencias en la tasa de TSS al comparar cuencas en una escala regional. Usualmente, se presentan altos valores de TSS en sustratos relativamente no consolidados y fácilmente erosionables (es decir, sedimentos cenozoicos del Plioceno al Eoceno), y bajos valores en formaciones resistentes (rocas plutónicas del Jurásico y el Cretácico), lo que resalta la importancia de los factores geológicos al determinar la magnitud de los procesos erosivos (Inman \& Jenkins, 1999).

En síntesis, la capacidad de almacenamiento de una cuenca, los tiempos de rezago y sus características litológicas dan lugar a que algunas cuencas de drenaje presentan una respuesta lenta o invariable frente a los cambios ambientales. En otras cuencas se produce el efecto contrario. En algunos casos, estos procesos pueden crear un desacoplamiento entre los cambios que se experimentan en la parte alta 0 media de una cuenca y los que se reflejan en su parte inferior (Phillips, et al., 2004). Por todo lo anterior, consideramos que los mecanismos forzadores climáticos y oceanográficos asociados a las oscilaciones detectadas mediante el análisis espectral definen un marco de variabilidad de mediano a largo plazo del TSS, mientras que las características fisiográficas, litológicas y ambientales de la cuenca definen la capacidad de magnificar, atenuar o desplazar temporalmente estas señales.

\section{Consideraciones sobre las fuentes de incertidumbre}

Es relevante delimitar el alcance de los resultados de este trabajo considerando las fuentes de incertidumbre asociadas a ellos y a su interpretación. La incertidumbre se relaciona, principalmente, con los métodos de estimación del TSS y el desfase que existe en la escala espacial y temporal de los procesos hidrológicos y geológicos. El IDEAM estima el TSS mediante la utilización de curvas de calibración, utilizando datos de mediciones simultáneas de nivel, caudal y concentración de sedimentos en suspensión durante diferentes condiciones de caudal (altos, medios y bajos). A pesar de que esta estimación se hace mediante un procedimiento estándar, se ha planteado que la inexactitud de los valores de SSL puede ser hasta del 7 \% (IDEAM,
2013). Además, a pesar de que las series de tiempo evaluadas eran relativamente largas, considerando la alta variabilidad natural del TSS consideramos que se requieren series más extensas para estimar promedios de largo plazo más confiables y, especialmente, para evaluar la importancia relativa de las oscilaciones a largo plazo en la variabilidad del TSS.

Debido a las diferencias que existen entre los procesos geológicos (es decir, la producción de sedimentos) e hidrológicos (o sea el transporte de sedimentos), no existe continuidad en la transferencia de sedimentos desde las áreas de fuente hasta los sistemas fluviales y, por lo tanto, hasta las desembocaduras. La magnitud de este desfase puede estimarse mediante el SDR (Walling, 1983). En la interpretación de los resultados se consideró que las cuencas con áreas de drenaje es menor a $5.000 \mathrm{~km}^{2}$ presentan altos valores de SDR, mientras que las cuencas con áreas de drenaje mayores a $14.000 \mathrm{~km}^{2}$ presentan bajos valores de SDR. Aun cuando esta decisión tuvo un sustento teórico válido (Walling, 1983; Dearing \& Jones, 2003), no se contaba con datos que permitieran conocer el valor específico de la SDR de los ríos evaluados en este estudio.

\section{Conclusiones}

Con un TSS acumulado de 146,3 x106 $\mathrm{t} \mathrm{a}^{-1}$, los ríos evaluados en este estudio contribuyeron en el periodo de estudio aproximadamente con el $39 \%$ de la descarga total de sedimentos estimada para la zona litoral del mar Caribe. El río Magdalena contribuyó con la mayor cantidad de estos sedimentos, con un TSS de 142,6 x10 $\mathrm{ta}^{-1}$, que correspondió al $38 \%$ del total de los aportes de sedimentos en suspensión estimados para esta región. Se ha considerado que la ausencia de tendencias significativas de TSS en la mayoría de estos ríos se debe fundamentalmente a su alta de variabilidad natural y a los cambios con efectos opuestos en los procesos de transporte de sedimentos. El análisis resaltó que después del año 2000 la mayoría de las tendencias del TSS cambiaron y reflejaron los cambios experimentados por estos sistemas durante el periodo 2000-2010 en sus tasas anuales de TSS, las cuales registraron variaciones hasta de $36 \%$.

Al comparar los espectros globales de ondícula del caudal y del TSS se observaron resultados similares en cuanto a la detección de las principales componentes oscilatorias. También se encontró que, aunque las oscilaciones dominantes del TSS no estuvieron en fase, en algunos de los ríos sus periodos de máxima potencia mostraron una relativa proximidad temporal. Estos resultados nos permitieron sugerir que los mecanismos forzadores climáticos u oceanográficos asociados a las oscilaciones detectadas mediante el análisis espectral definen un marco de variabilidad de mediano a largo plazo del TSS, mientras que las características fisiográficas y litológicas de las cuencas definen la capacidad de magnificar, atenuar o desplazar temporalmente estas señales. En la mayoría de estos ríos 
el análisis espectral de ondícula evidenció la presencia simultánea de varias oscilaciones en periodos en los que se observaron altas tasas de TSS, como fueron los de 19871990, 1994-2002 y 2008-2010.

\section{Agradecimientos}

Esta investigación fue financiada por la Universidad del Norte (Dirección de Investigación, Desarrollo e Innovación, DIDI). Se agradece especialmente el apoyo del programa de becarios de la Universidad del Norte y la beca del CEMarin (Centro de Excelencia para las Ciencias del Mar, financiado por el Servicio Alemán de Intercambio Académico, DAAD), otorgada a uno de los autores (J.C. Restrepo). Así mismo, los autores agradecen los valiosos aportes hechos por los evaluadores, los cuales permitieron mejorar la calidad del manuscrito.

\section{Conflicto de intereses}

Los autores manifiestan que no tienen conflicto de intereses.

\section{Bibliografía}

Alvarado, M. 1992. Río Magdalena (sector Calamar-Bocas de Ceniza): caracterización hidrosedimentológica y cuña salina basada en mediciones realizadas durante 20 campañas. Reporte Técnico, Ministerio de Obras Públicas y Transporte, Barranquilla, Colombia.

Bernal, G., Poveda, G., Roldan, P., Andrade, C. 2006. Patrones de variabilidad de las temperaturas superficiales del mar en la costa Caribe colombiana. Revista de la Academia Colombiana de Ciencias Exactas, Físicas y Naturales. 30 (115): 195-208.

Cediel, F., Shaw, R., Caceres, M. 2003. Tectonic assembly of the Northern Andean Block. En: Bartolini, C., Buffler, R., Blickwede, J. (Eds.) The circum-gulf of Mexico and Caribbean Hydrocarbon habitats, basin formation and plate tectonics. AAPG Memoir, 79 p.

Dai, A., Qian, T., Trenberth, K., Milliman, J. 2009. Changes in continental freshwater discharge from 1948 to 2004. Journal of Climate. 22: 2773-2792.

Dearing, J., Jones, R. 2003. Coupling temporal and spatial dimensions of global sediment flux through lake and marine sediment records. Global and Planetary Change. 39: $147-168$.

Duque-Caro, H. 1979. Major structural elements and evolution of northwestern Colombia. American Association of Petroleum Geologist Memoir. 29: 329-351.

Duque-Caro, H. 1984. Estilo estructural, diapirismo y episodios de acrecimiento del terreno Sinú-San Jacinto en el noroccidente de Colombia. Boletín Geológico del Ingeominas. 27 (2): 29 p.

Dyer, K. 1995. Sediment Transport Processes in Estuaries. En: Perillo G.M.E. Geomorphology and Sedimentology of Estuaries. Developments in Sedimentology. Elsevier Science. 53: 423-449.

Dyer, K. 1997. Estuaries: A Physical Introduction. Jhon Wiley, Second Edition. Chichester, England 195 p.
Etter, A., McAlpine C., Wilson, K., Phinn, S., Possingham, H. 2006. Regional patterns of agricultural land use and deforestation in Colombia. Agriculture Ecosystems \& Environment. 114: 369-386.

Fan, H., Huang, H. 2005. Changes in Huanghe (Yellow) River estuary since artificial re-routing in 1996. Chinese Journal of Oceanology and Limnology. 23 (9): 299-305.

García, N., Mechoso, C. 2005. Variability in the discharge of South American rivers and in climate. Journal of Hydrological Sciences. 50 (3): 459-478.

Gao, S., Wang, Y., Gao, J. 2011. Sediment retention at the Changjiang sub-aqueous delta over a 57 year period, in response to catchment changes. Estuarine, Coastal and Shelf Science. 95: 29-38.

Harrison, C.G. 2000. What factors control mechanical erosion rates. Int. Journal of Earth Sciences. 88: 1-11.

Hovius, N. 1998. Controls on sediment supply by large rivers. SEPM Special Publication. 59: 3-16.

Instituto de Hidrologia, Meteorología y Estudios Ambientales - Colombia. 1998. El medio ambiente en Colombia. IDEAM, Bogotá, 495 p.

Instituto de Hidrología, Meteorología y Estudios Ambientales - Colombia. 2013. Lineamientos conceptuales y metodológicos para la evaluación regional del agua. IDEAM, Bogotá, 275 p.

Ingeominas. 2003. Geología de los cinturones Sinú-San Jacinto. Planchas 50 Puerto Escondido, 51 Lorica, 59 Mulatos, 60 Canalete, 61 Montería, 69 Necoclí, 70 San Pedro de Urabá, 71 Planeta Rica, 79 Turbo, 80 Tierralta. Escala 1:100.000.

Inman, D., Jenkins, S. 1999. Climate change and the episodicity of sediment flux of small California rivers. The Journal of Geology. 107: 251-270.

Kettner, A., Restrepo, J.D., Syvitski, J.P.M. 2010. A spatial simulation experiment to replicate fluvial sediment fluxes within the Magdalena River basin, Colombia. The Journal of Geology. 118: 363-379.

Labat, D. 2005. Recent advances in wavelet analyses: Part 1 - A review of concepts. Journal of Hydrology. 314: 275-288.

Labat, D., Ronchall, J., Guyot, J.L. 2005. Recent advances in wavelet analyses: Part: 2 - Amazon, Paraná, Orinoco and Congo discharges time scale variability. Journal of Hydrology. 314: 289-311.

Le, T., Garnier, J., Gilles, B., Sylvain, T., Minh, Ch. 2007. The changing flow regime and sediment load of the Red River, Viet Nam. Journal of Hydrology. 334: 199-214.

Liquete, C., Canals, M., Ludwig, W., Arnau, P. 2009. Sediment discharge of the rivers of Catalonia, NE Spain, and the influence of human impacts. Journal of Hydrology. 366: 76-88.

Liu, Q.; Shi, Z.; Fang, N.; Zhu, H.; Ai, L. 2013. Modeling the daily suspended sediment concentration in a hyperconcentrated river on the Loess Plateau, China, using the Wavelet-ANN approach. Geomorphology. 186: 181-190. 
Kettner, A.J., Restrepo, J.D., Syvitski, J.P.M. 2010. A spatial simulation experiment to replicate fluvial sediment fluxes within the Magdalena River basin, Colombia. Journal of Geology. 118: 363-379.

Marín, R. 1992. Estadísticas sobre el recurso agua en Colombia. Ministerio de Agricultura - Instituto Colombiano de Hidrología, Meteorología y Adecuación de Tierras. 2 Edición. Bogotá.

Martínez, J.I., López, E. 2005. El sistema fluvial del Magdalena durante el Plioceno-cuaternario: contexto tectónico y escenario paleoclimático. En: Restrepo, J.D. (Ed.): Los sedimentos del río Magdalena: reflejo de la crisis ambiental. Fondo Editorial Universidad Eafit, Medellin, Colombia. p. 101-112.

Meade, R. 1996. River-sediment inputs to major deltas. In: Milliman, J.D., and Haq, B.U. (Eds.). Sea level rise and coastal subsidence. Dordrecht, Kluwer, p. 63-85.

Mesa O., Poveda G., Carvajal L. 1997. Introducción al clima de Colombia. Universidad Nacional de Colombia, Bogotá, Colombia. 390 p.

Meybeck, M., Laroche, L., Durr, H., Syvitski, J. 2003. Global variability of daily total suspended solids and their fluxes in rivers. Global and Planetary Change. 39: 65-93.

Milliman, J. D., Meade, R. H. 1983. World-wide delivery of river sediment to the oceans. Journal of Geology. 91: 1 - 21.

Milliman, J. D., Syvitski, P.M. 1992. Geomorphic/Tectonic control of sediment discharge to the ocean: The importance of small mountainous rivers. Journal of Geology. 100: 525-544.

Milliman, J.D., Farnworth, K., Jones, P., Xu, K., Smith, L. 2008. Climatic and anthropogenic factors affecting river discharge to the global ocean, 1951-2000. Global and Planetary Change. 62: 187-194.

Mojica, J., Franco, R. 1990. Estructura y evolución tectónica del Valle Superior y Medio del Magdalena, Colombia. Geología Colombiana. 17: 41-64.

Montes, C., Guzmán, G., Bayona, G., Cardona, A., Valencia, V., Jaramillo, C. 2010. Clockwise rotation of the Santa Marta massif and simultaneous Paleogene to Neogene deformation of the Plato-San Jorge and Cesar-Rancheria basins. Journal of South American Earth Sciences. 29: 832848.

Morehead, M.D., Syvitski, J., Hutton, E., Peckam, S. 2003. Modeling the temporal variability in the flux of sediment from ungauged river basins. Global and Planetary Change. 39: 95-110.

Netherlands Engineering Consultants (NEDECO). 1973. Río Magdalena and Canal del Dique survey project. Technical Report. The Hague, The Netherlands. 397 p.

National Oceanic and Atmospheric Administration (NOAA). 2012. National Weather Service - Climate Prediction Center. United States. Base de datos en línea (http:// www.cpc.ncep.noaa.gov/products/analysis_monitoring/ ensostuff/ensoyears.shtml) (Diciembre, 2012).
Pasquini, A., Depetris, P. 2007. Discharge trends and flow dynamics of South American rivers draining the southern Atlantic seaboard: An overview. Journal of Hydrology. 333: 385-399.

Park, D. 1999. Waves, tides and shallow-water processes. Second edition. The Open University, Butterworth-Heinemann. London, United Kingdom. 227 p.

Phillips, J.D., Slattery, M.C., Musselman, Z.A. 2004. Dam-todelta sediment inputs and storage in the lower Trinity River, Texas. Geomorphology. 62: 17-34.

Poveda, G. 2004. La hidroclimatología de Colombia: una síntesis desde la escala inter-decadal hasta la escala diurna. Revista de la Academia Colombiana de Ciencias Exactas, Físicas y Naturales. 28 (107): 201-222.

Poveda, G., Jaramillo A., Gil M., Quiceno N., Mantilla R. 2001. Seasonality in ENSO-related precipitation, river discharges, soil moisture, and vegetation index in Colombia. Water Resources Research. 37 (8): 2169-2178.

Poveda, G., Mesa O. 2004. On the existence of Lloro (the rainiest locality on Earth): Enhanced ocean-atmosphereland interaction by a low-level jet. Geophysical Research Letters. 27 (11), 1675-1678.

Restrepo, J.C., Restrepo, J.D. 2005. Efectos naturales y antrópicos en la producción de sedimentos de la cuenca del río Magdalena. Revista de la Academia Colombiana de Ciencias Exactas, Físicas y Naturales. 29 (111): 239-254.

Restrepo, J.C., Ortíz, J.C., Pierini, J., Schrottke, K., Maza, M., Otero, L. 2014. Freshwater discharge into the Caribbean Sea from the Rivers of Northwestern South America (Colombia): Magnitude, variability and recent changes. Journal of Hydrology. 509: 266-281.

Restrepo J.D., Kjerfve B. 2000. Magdalena river: Interannual variability (1975-1995) and revised water discharge and sediment load estimates. Journal of Hydrology. 235: 137-149.

Restrepo, J.D., Kjerfve, B. 2004. The Pacific and Caribbean Rivers of Colombia: Water Discharge, Sediment Transport and Dissolved Loads. En: Lacerda, L.; Santelli, R.; Duursma, E.; Abrao, J., (Eds.). Environmental Geochemistry in Tropical and Subtropical Environments. Springer Verlag, Berlín, p. 169-187.

Restrepo, J.D., Kjerfve, B., Hermelín, M., Restrepo, J.C. 2006. Factors controlling sediment yield in a major South American drainage basin: the Magdalena River, Colombia. Journal of Hydrology. 316: 213-232.

Restrepo, J.D., López, S. 2008. Morphodynamics of the Pacific and Caribbean deltas of Colombia - South America. Journal of South American Earth Sciences. 25: 1-21.

Restrepo, J.D., Syvitski, J.P.M. 2006. Assessing the effect of natural controls and land use change on sediment yield in a major Andean River: The Magdalena drainage basin, Colombia. Ambio: a Journal of the Human Environment. 35: 44-53.

Shumway R., Stoffer D. 2004. Time Series Analysis and its Applications. Springer-Verlag, Amsterdam, The Netherlands. 549 p. 
Slattery, M., Phillips, J. 2009. Controls on sediment delivery in coastal plain rivers. Journal of Environmental Management. 92: 284-289.

Syvitski, J.P.M. 2011. Global sediment fluxes to the Earth's coastal ocean. Applied Geochemistry. 26: S373-S374.

Syvitski, J.P.M., Kettner, A., J. 2011. Sediment flux and the Anthropocene. Philosophical Transactions of the Royal Society A Mathematical, Physical and Engineering Sciences. 369 (1938): 957-975.

Torrence C., Compo G. 1998. A practical guide to wavelet analysis. Bulletin of the American Meteorological Society. 79 (1): 61-78.

Trenkamp, R., Kellog, J., Freymueller, J., Mora, H. 2002. Wide plate margin deformation, southern Central America and northwestern South America, CASA GPS observations. Journal of South American Earth Sciences. 15 (2): 157-171.

Van der Hammen, T. 1986. Fluctuaciones holocénicas del nivel de inundaciones en la cuenca del bajo Magdalena-CaucaSan Jorge (Colombia). Geología Norandina. 10: 11-17.

Walling, D. 1983. The sediment delivery problem. Journal of Hydrology. 65: 209-225.

Walling, D. 2009. The impact of global change on erosion and sediment transport by rivers: Current progress and future challenges. The United Nations World Water Development Report 3: Water in a Changing World. Scientific Paper. UNESCO, Paris, France. 26 p.
Walling, D., Fang, D. 2003. Recent trends in the suspended sediment loads of the world's rivers. Global and Planetary Change. 39: 111-126.

Wang, H., Bi, N., Saito, Y., Wang, Y., Sun, X., Zhang, J. 2010. Recent changes in sediment delivery by the Huanghe (Yellow River) to the sea: Causes and environmental implications in its estuary. Journal of Hydrology. 391: 302-313.

Wang, H., Yang, Z., Saito, Y., Liu, P., Sun, X., Wang, Y. 2007. Stepwise decreases of the Huanghe (Yellow River) sediment load (1950-2005): Impacts of climate change and human activities. Global and Planetary Change. 57: 331-354.

Wilkinson, B.H. 2005. Human as geologic agents: A deep-time perspective. Geology. 33: 161-164.

Yang, S., Belkin, I., Belkina, A., Zhao, Q., Zhu, J., Ding, P. 2003. Delta response to decline in sediment supply from the Yangtze River: Evidence of the recent four decades and expectations for the next half-century. Estuarine, Coastal and Shelf Science. 57: 689-699.

Yue S., Pilon, P., Cavadias, G. 2002. Power of the Mann-Kendall and Spearman's rho test to detecting monotonic trends in hydrological series. Journal of Hydrology.259: 254-271.

Zhang, J., Wei, F., Liu, S. 2008. Possible effect of ENSO on annual sediment discharge of debris flow in the Jiangjia Ravine based on Morlet wavelet transforms. International Journal of Sediment Research. 23 (3): 267-274. 